\title{
The Importance of the Precipitation Mass Sink in Tropical Cyclones and Other Heavily Precipitating Systems
}

\author{
GARY M. LACKMANN AND RICHARD M. YABLONSKY \\ Department of Marine, Earth, and Atmospheric Sciences, North Carolina State University, Raleigh, North Carolina
}

(Manuscript received 22 July 2003, in final form 8 January 2004)

\begin{abstract}
When water vapor is converted to cloud and precipitation and subsequently removed to the surface via precipitation, there is a corresponding hydrostatic pressure decrease due to the reduction of mass in the overlying column. Pressure changes resulting from the addition or removal of water vapor are currently neglected in most meteorological applications. However, in heavily precipitating systems such as tropical cyclones, where precipitation rates may exceed $250 \mathrm{~mm}$ day $^{-1}$, the pressure equivalent of the precipitation mass sink is not negligible $\left(\sim 25 \mathrm{hPa}\right.$ day $\left.^{-1}\right)$. Pressure decreases due to this mechanism are most pronounced in the lower troposphere, particularly below the melting level. The resulting unbalanced pressure-gradient force can enhance convergence, which precludes full realization of the pressure decrease but may contribute to vorticity generation and moisture convergence.

The importance of the precipitation mass sink is investigated for the case of Hurricane Lili (2002) through the computation of mass and potential vorticity $(\mathrm{PV})$ budgets and numerical sensitivity experiments. The precipitation mass reaching the surface within $100 \mathrm{~km}$ of the storm center is of the same order as the mass loss needed to explain the area-averaged pressure decrease during the intensification stage of Lili. The PV is altered by precipitation mass flux divergence across isentropic layers. A volume-integrated PV budget reveals that the mass sink term is small in comparison to the latent heating term, although the latter exhibits large cancellation. Comparison of a control simulation from the Eta Model to an experimental simulation in which the precipitation mass sink effect is included demonstrates that the mass sink mechanism contributes to lower pressure, stronger wind speeds, and heavier precipitation. The sea level pressure near the storm center in the mass sink simulation is generally $2-5 \mathrm{hPa}$ deeper relative to the control simulation, with $10-\mathrm{m}$ wind speed differences of 5 to $15 \mathrm{kt}$. The mass sink simulation exhibits a stronger cyclonic PV tower, especially above the melting level, and a stronger troposphere-deep cyclonic circulation relative to the control simulation. The analysis presented indicates that the precipitation mass sink mechanism, though not dominant, is not negligible for tropical cyclones.
\end{abstract}

\section{Introduction}

When water vapor is converted to cloud and precipitation, there is a corresponding decrease in atmospheric mass. ${ }^{1}$ If the condensate is subsequently removed to the surface via precipitation, a hydrostatic pressure decrease occurs due to the reduction of mass in the overlying air column. Likewise, evaporation of water vapor from the surface provides a slight increase in the hydrostatic pressure. Most numerical, dynamical, and conceptual models of the atmosphere neglect these mass transfer processes (Gu and Qian 1991; Qiu et al. 1993), presumably because the mass of water vapor lost (gained) during condensation, and deposition (evaporation and subli-

\footnotetext{
${ }^{1}$ Our definition of the atmosphere is restricted to the gaseous portion, exclusive of cloud and precipitation material.
}

Corresponding author address: Dr. Gary M. Lackmann, Dept. of Marine, Earth, and Atmospheric Sciences, North Carolina State University, Raleigh, NC 27695-8208.

E-mail: gary@ncsu.edu mation) is usually negligible in comparison to other transfer mechanisms. However, in the vicinity of heavy precipitation this assumption is questionable. The pressure equivalent of $25 \mathrm{~mm}$ of liquid precipitation can be computed by multiplying the water depth $(0.025 \mathrm{~m})$ by the density of liquid water $\left(1000 \mathrm{~kg} \mathrm{~m}^{-3}\right)$ and the gravitational acceleration $\left(9.8 \mathrm{~m} \mathrm{~s}^{-2}\right)$. We find that the pressure equivalent of $25 \mathrm{~mm}$ of precipitation is about 2.5 $\mathrm{hPa}$. For tropical cyclones, in which precipitation rates of $250 \mathrm{~mm}$ day $^{-1}$ or more have been observed, the corresponding pressure-equivalent mass depletion would be $25 \mathrm{hPa}$ or more, and a variety of positive feedback scenarios emerge for consideration. Of course, we would not expect the full hydrostatic pressure decrease associated with the precipitation mass sink to be realized because horizontal convergence arises in response to the unbalanced pressure-gradient force. But it is precisely this compensating inflow that may be critical to moisture transport and vorticity generation, especially in the presence of strong rotation. The hypothesis advanced here is that the hydrostatic pressure reduction due to the re- 
moval of atmospheric mass by precipitation exerts a nonnegligible influence on the dynamics of tropical cyclones and other heavily precipitating cyclonic systems. It is further hypothesized that inclusion of the mass sink mechanism in numerical models will produce lower central pressure, stronger cyclonic wind speeds, and enhanced precipitation.

Although modern numerical weather prediction (NWP) models include water substance continuity, most do not explicitly account for the precipitation mass sink effect in the pressure-tendency or full-continuity equations $^{2}$ (Gu and Qian 1991; Qiu et al. 1991, 1993; Davies et al. 2002). Even sophisticated mesoscale models such as the operational version of the National Centers for Environmental Prediction (NCEP) Eta Model and the fifth-generation Pennsylvania State University-National Center for Atmospheric Research (PSU-NCAR) Mesoscale Model (MM5) do not explicitly account for the precipitation mass sink (F. Mesinger and J. Dudhia 2002, 2003, personal communication). The neglect of the precipitation mass sink in NWP models has been questioned by Gu and Qian (1991), Qiu et al. (1991, 1993), Van den Dool and Saha (1993), and more recently by Davies et al. (2002). Gu and Qian (1991) performed a scale analysis of the surface-pressure tendency equation and determined that when the rainfall rate exceeds $\sim 25$ $\mathrm{mm}$ day $^{-1}$ the precipitation mass sink becomes nonnegligible. Qiu et al. (1993) analyzed a rather typical extratropical cyclone in the eastern United States and found that an overall increase in precipitation amount resulted when the mass sink effect was explicitly included in a numerical model, with local precipitation increases of greater than $20 \mathrm{~mm}$ in a 36-h forecast. Savijärvi (1995) ran one-dimensional simulations with a sigma coordinate model and found that although vertical velocity changes due to "water mass forcing" were small, surface pressure changes were significant, exhibiting diurnal fluctuations due to the mass source/sink on the order of several hectopascals per day.

As water vapor and cloud condensate are removed in the form of precipitation from the middle and lower troposphere, the mass sink effect will lead to local pressure falls and the corresponding development of an unbalanced pressure-gradient force directed toward the region of maximum mass removal. The resulting convergent inflow would be strongest in the lower troposphere, as depicted schematically in Fig. 1. In the lower troposphere below the level of maximum latent heat release from condensation and deposition, the pressure tendency due to the mass sink acts in the same sense as that associated with the latent heating. Pressure changes attributable to latent heat release are well documented and would be expected to dominate those due to the precipitation mass sink effect. However, a direct compar-

\footnotetext{
${ }^{2}$ Some exceptions appear to include the Weather Research and Forecasting (WRF) model, the recent model developed by Ooyama (2001), and an experimental version of the Eta Model presented here.
}

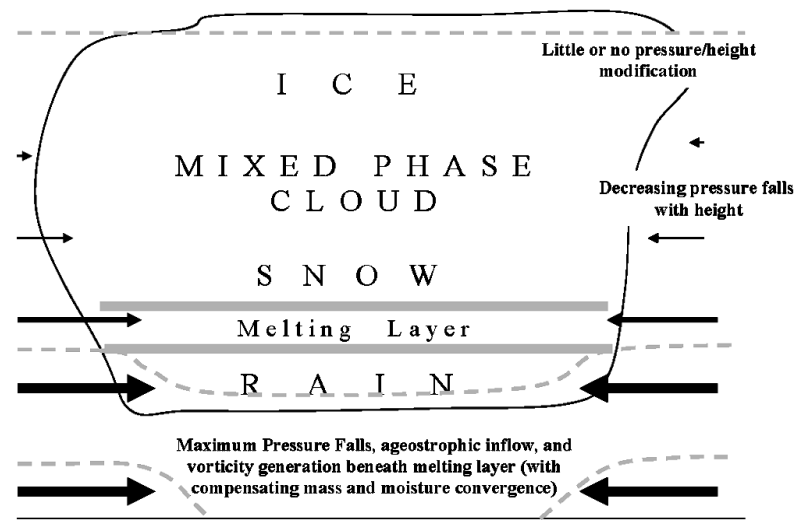

FIG. 1. Schematic diagram representing a heavily precipitating system, including implied height surfaces (dashed gray lines) and compensating horizontal ageostrophic motion (arrows) arising in response to the precipitation mass sink.

ison of these two mechanisms is complicated by the fact that the net heating due to latent heat release is dependent upon the environmental lapse rate. In a moist-adiabatic environment, the heating provided by latent heat release is exactly cancelled by adiabatic expansion. Therefore, the net heating (and surface pressure fall) can be small even with heavy precipitation, and it is often determined by the small difference between large terms in the thermodynamic equation (e.g., Anthes 1982, his section 2.2).

The objective of this paper is to revisit the assumption that the precipitation mass sink is negligible for heavily precipitating systems. Our hypothesis is that the mass sink effect is not negligible. Three primary tests of this hypothesis are conducted for the case of Hurricane Lili (2002), including (i) a mass budget, (ii) a potential vorticity (PV) budget, and (iii) sensitivity experiments using control and experimental versions of a numerical weather prediction model. The remainder of this paper is organized as follows. Section 2 outlines the basic equations and methodology, section 3 discusses methodology relating to the numerical models used in this study, section 4 presents results of the hypothesis tests for the case of Hurricane Lili, and section 5 contains a concluding discussion and future research directions.

\section{Equations and methodology}

\section{a. The continuity equation}

The most conspicuous appearance of the precipitation mass sink is in the mass continuity equation. In most meteorological applications, this mass conservation relation is written without account of source and sink terms; in isobaric coordinates, the continuity equation is often written in the convenient form

$$
\boldsymbol{\nabla} \cdot \mathbf{v}+\frac{\partial \omega}{\partial p}=0
$$


where $\mathbf{v}$ is the horizontal velocity vector and $\boldsymbol{\nabla}$ is the horizontal gradient operator. As noted by Trenberth (1991), a more accurate form of (1) is

$$
\boldsymbol{\nabla} \cdot \mathbf{v}+\frac{\partial \omega}{\partial p}=E-P,
$$

where $E$ and $P$ represent evaporation and precipitation source and sink terms, respectively. Trenberth (1991) notes that in a given atmospheric layer, the right-hand side of (2) is typically negligible. However, Trenberth also notes that when (2) is integrated vertically, the relative importance of the right side increases owing to strong cancellation in the left-hand terms, with no such cancellation in $E-P$. In studies involving global mass budgets, the water vapor source/sink term has been shown to play a significant role in hemispheric pressure fluctuations (e.g., Trenberth et al. 1987; Trenberth 1991; Van den Dool and Saha 1993).

Several earlier studies have considered the source and sink terms in the continuity equation. For example, Dutton $(1986$, his section 8.1$)$ writes the moist-air continuity equation as

$$
\boldsymbol{\nabla}_{3} \cdot \mathbf{u}=-\frac{1}{\rho_{m}} \frac{d \rho_{m}}{d t}+\frac{1}{1-q} \frac{d q}{d t},
$$

where $\mathbf{u}$ denotes the three-dimensional wind vector, $\rho_{m}$ is the mass density of moist air (dry air plus water vapor), $\boldsymbol{\nabla}_{3}$ is the three-dimensional gradient operator, and $q$ is the specific humidity. Also, Gu and Qian (1991) and Qiu et al. (1993) present sigma-coordinate forms of the continuity equation containing the precipitation mass source/sink terms. Recent studies by Ooyama $(1990,2001)$ and Schubert et al. (2001) provide an advanced framework for atmospheric dynamics that is designed for application to moist, nonhydrostatic convection. These equations include explicit representation of precipitation mass source/sink terms. The continuity equation in this system ${ }^{3}$ is given by

$$
\frac{\partial \rho_{t}}{\partial t}+\boldsymbol{\nabla} \cdot\left(\rho_{t} \mathbf{v}\right)+\frac{\partial\left(\rho_{t} w+\rho_{r} W\right)}{\partial z}=0,
$$

where $w$ is the vertical velocity, $\rho_{t}=\rho_{a}+\rho_{v}+\rho_{c}+$ $\rho_{r}$ is the total mass density (sum of dry air, water vapor, cloud condensate, and precipitation), $\rho_{r}$ is the mass density of precipitation, and $W$ is the hydrometeor terminal fall speed. Computations of the terms in (4) for a heavily precipitating tropical cyclone indicate that the precipitation flux term is approximately two orders of magnitude smaller than the leading terms in the equation (not shown), which is sufficient cause to justify neglect of this process in most applications. However, in light of the aforementioned observations of Trenberth (1991),

\footnotetext{
${ }^{3}$ The precipitation terms also arise in the momentum and thermodynamic equations, in order to include momentum and entropy transferred by falling precipitation.
}

it does not follow that the mass sink is negligible in the pressure-tendency equation.

\section{b. The pressure-tendency equation}

Trenberth (1991) provides a surface-pressure tendency equation that includes precipitation and evaporation sink/source terms in isobaric coordinates [his Eq. (5)], and both $\mathrm{Gu}$ and Qian (1991) and Qiu et al. (1993) present the complete pressure-tendency equation in sigma coordinates. The local hydrostatic surface-pressure tendency due to the removal of mass from precipitation reaching the surface is given by

$$
\left.\frac{\partial p_{\text {sfc }}}{\partial t}\right|_{\text {water mass transfer }}=-g \rho_{\ell} R+g \rho_{\ell} E,
$$

where $\rho_{\ell}$ is the density of liquid water, $R$ is the surface liquid-equivalent precipitation rate (in $\mathrm{m} \mathrm{s}^{-1}$ ), and $E$ is the liquid-equivalent evaporation rate (in $\mathrm{m} \mathrm{s}^{-1}$ ). For the mass budget presented in section 4, a storm-relative pressure-tendency equation for a storm-centered cylindrical volume of $100-\mathrm{km}$ radius is utilized,

$$
\left(\frac{d \bar{p}_{\text {sfc }}}{d t}\right)_{\text {storm }}=\frac{1}{A} \int_{0}^{H_{\mathrm{top}}} \oint g \rho u_{r} d s d z-g \rho_{\ell} \bar{R},
$$

where $u_{r}$ is the radial wind component, $\bar{p}_{\text {sfc }}$ is the average sea level pressure within the cylinder, $A$ is the area of the cylinder, and we neglect evaporation from the sea surface within the cylinder (see, e.g., Palmén and Riehl $1957,{ }^{4}$ their section 6). The first right-hand term in (6) is the lateral mass flux across the cylinder boundary, and it is assumed that the mass flux out of the cylinder top (taken here to be the $100-\mathrm{hPa}$ level) is negligible.

When computing terms in (6) from numerical model output, there is difficulty in comparing terms computed from instantaneous fields (the first right-hand term) with those based on hourly time differencing (the left side and second right side terms). Models such as MM5 do not explicitly account for the precipitation mass sink in the pressure-tendency equation, so the local model pressure tendency will not account for pressure reduction due to the mass sink (the second right-hand term). However, in the nonhydrostatic version of MM5 used here, water loading (and water unloading when precipitation reaches the surface) may partially account for the precipitation effect (J. Dudhia 2002, personal communication). Therefore, in (6) we will simply compare the second right-side term to the left side of the equation. A more complete method of accounting for the mass sink effect on pressure will be obtained using results from a modified version of the NCEP Eta Model that explicitly accounts for the precipitation mass sink. Com-

\footnotetext{
${ }^{4}$ Interestingly, Palmén and Riehl (1957, p. 158) indirectly acknowledge the precipitation mass sink stating, "It follows that both from heat and moisture balance considerations the hurricane must be regarded as an open system.",
} 
parison of simulations from this version of the model with a control simulation will be used to provide a quantitative evaluation of the importance of the mass sink.

As will be shown in section 4 for Hurricane Lili, the pressure-equivalent mass sink contribution is not negligible, especially in the hurricane eyewall region. However, the total pressure reduction due to the mass sink would not be realized because the unbalanced pressure gradient that would arise in response to the pressure fall would drive compensating horizontal mass convergence. The degree of compensation is a function of the Rossby radius of deformation, which depends on the background vorticity, the spatial scale of the pressurefall region, and the static stability. As noted by Ooyama (1982), the effective Rossby radius decreases in the strongly rotational environment of a tropical cyclone, meaning that a larger fraction of the precipitation mass sink pressure reduction would be realized in a hurricane relative to systems characterized by smaller vorticity. In Ooyama's terminology, the mesoscale environment is "stiffened" by the increased rotation, and the effective inertial stability is as much as two orders of magnitude higher in a mature hurricane relative to the surrounding tropical atmosphere. Depending on the scale and intensity of the precipitation mass sink, as well as the strength of preexisting vorticity, one must consider the potential for mass-sink-induced vorticity generation through the convergence term in the vorticity equation. For a developing tropical system that is characterized by heavy rainfall, a feedback involving the mass sink is possible as convergent inflow is increasingly effective in vorticity generation.

\section{c. The vorticity equation}

A form of the vorticity equation applicable near the surface (where $w \approx 0$ ) in height coordinates is

$$
\frac{d \zeta_{a}}{d t}=-\zeta_{a}(\boldsymbol{\nabla} \cdot \mathbf{v})+\mathbf{k} \cdot(\boldsymbol{\nabla} p \times \boldsymbol{\nabla} \alpha)+\mathbf{k} \cdot\left(\boldsymbol{\nabla} \times \mathbf{F}_{r}\right)
$$

where $\mathbf{F}_{r}$ represents friction. The efficiency of the convergence term [the first right-hand term in (7)] is proportional to the vorticity itself. Therefore, we anticipate that vorticity generation resulting from convergence in response to precipitation-induced pressure falls will be most important for strongly rotating systems. The convergence term in (7) is recognized as a dominant vorticity-generation mechanism, yet it has proven difficult to isolate and quantify the various physical processes that are the ultimate cause of the convergence. Several mechanisms can contribute to convergence in the nearsurface layer for a hurricane, including (i) friction, (ii) inflow arising in response to adiabatic processes aloft, (iii) response to pressure falls driven by latent heat release, and (iv) response to pressure falls driven by the precipitation mass sink. Earlier studies have considered the physical processes responsible for this inflow; fric- tion is known to contribute strongly to radial inflow in the boundary layer of hurricanes (e.g., Palmén and Riehl 1957; Charney and Eliassen 1964; Anthes 1982, his section 3.1). The unbalanced, inward-directed pressuregradient force generated by the precipitation mass sink would act to reinforce the convergent inflow, perhaps providing a nonnegligible contribution to the generation of vorticity through the convergence term. There are several options for isolation of this mechanism in the vorticity equation, including use of Ooyama's continuity equation (4)

$$
\boldsymbol{\nabla} \cdot \mathbf{v}=-\left(\frac{1}{\rho_{t}} \frac{d \rho_{t}}{d t}+\frac{\partial w}{\partial z}+\frac{1}{\rho_{t}} \frac{\partial \rho_{r} W}{\partial z}\right),
$$

and direct substitution into (7). Equation (8) reveals that the divergence is partially due to the vertical precipitation mass flux divergence; unfortunately, there are practical difficulties relating to the computation of (8), including a lack of information concerning the terminal hydrometeor fall speed $W$ and the discontinuous nature of the precipitation mass flux at the surface (see Ooyama 2001, his section 2c).

An alternative approach is to compute the isallobaric convergence (including that part arising from the precipitation mass sink) and then calculate the contribution of this term directly in the vorticity equation. However, as Sutcliffe (1947) notes, "... the rate of change of (vorticity) is related with the field of the development index $d p / d t$, but not in any simple manner.' Keyser and Johnson (1984) have presented related developments. In general, the vorticity tendency due to isallobaric convergence is proportional to the Laplacian of the local pressure tendency, a result shown by Sutcliffe (1947) and others. For the precipitation mass sink, we find accordingly that the term is proportional to the Laplacian of the precipitation rate. This relationship indicates that the most efficient vorticity generation would accompany concentrated regions of heavy rainfall in the presence of strong rotation.

\section{d. The potential vorticity equation}

As water vapor and cloud material originating in a given isentropic layer is converted to precipitation that falls below the isentropic surface defining the bottom of the layer, "PV substance" (also known as isentropic absolute vorticity) is concentrated within the layer, leading to a PV increase there (e.g., Haynes and McIntyre 1987). Schubert et al. (2001) present the PV-tendency equation for the equations of Ooyama (2001), including a term arising from the precipitation mass sink:

$$
\frac{d P}{d t}=\frac{1}{\rho_{t}}\left[\left(\boldsymbol{\nabla}_{3} \times \mathbf{F}_{r}\right) \cdot \nabla_{3} \theta_{\rho}+\boldsymbol{s} \cdot \nabla_{3} \dot{\theta}_{\rho}+P \boldsymbol{\nabla}_{3} \cdot\left(\rho_{r} \mathbf{U}\right)\right]
$$

Here, $P=\left(1 / \rho_{t}\right) \boldsymbol{s} \cdot \nabla_{3} \theta_{\rho}$ is the potential vorticity, $\theta_{\rho}=\left(p / \rho_{t} R_{\text {air }}\right)\left(p_{0} / p\right)^{\kappa}, \boldsymbol{s}$ is the absolute vorticity vector 
$2 \Omega+\boldsymbol{\nabla} \times \mathbf{u}, \dot{\theta}_{\rho}$ is the material derivative of $\theta_{\rho}$, and $\mathbf{U}$ is the velocity of precipitation relative to the air. The three terms on the right side of (9) can be separated as follows:

$$
\begin{aligned}
\left.\frac{d P}{d t}\right|_{\text {friction }} & =\frac{1}{\rho_{t}}\left[\left(\nabla_{3} \times \mathbf{F}_{r}\right) \cdot \boldsymbol{\nabla} \theta_{\rho}\right], \\
\left.\frac{d P}{d t}\right|_{\text {diabatic }} & =\frac{1}{\rho_{t}}\left(\boldsymbol{s} \cdot \nabla_{3} \dot{\theta}_{\rho}\right) \\
& =\frac{1}{\rho_{t}}\left(\zeta_{x} \frac{\partial \dot{\theta}_{\rho}}{\partial x}+\zeta_{y} \frac{\partial \dot{\theta}_{\rho}}{\partial y}+\zeta_{z} \frac{\partial \dot{\theta}_{\rho}}{\partial z}\right), \\
\left.\frac{d P}{d t}\right|_{\text {mass sink }} & =\frac{1}{\rho_{t}}\left[P \nabla_{3} \cdot\left(\rho_{r} \mathbf{U}\right)\right] \\
& =\frac{1}{\rho_{t}}\left[P \frac{\partial}{\partial z}\left(\rho_{\mathrm{ra}} W_{\mathrm{ra}}+\rho_{\mathrm{sn}} W_{\mathrm{sn}}+\rho_{\mathrm{gr}} W_{\mathrm{gr}}\right)\right] .
\end{aligned}
$$

We are most interested in the relative magnitude of the diabatic term to the mass sink term, and hence we will only evaluate (11) and (12). In order to obtain the diabatic contribution (11), MM5 (Dudhia 1993; Grell et al. 1994) was modified to output individual terms in the model temperature-tendency equation, including the tendencies attributable to (i) grid-scale latent heat release or absorption, (ii) the cumulus parameterization scheme, (iii) the planetary boundary layer scheme, (iv) the radiation scheme, and (v) diffusion. The heating rate $\dot{\theta}_{\rho}$ was computed as the sum of only the grid-scale and cumulus parameterization contributions, consistent with our focus on precipitation processes. The latent heating rates were checked against the parameterization presented by Emanuel et al. (1987) and were found to exhibit a high degree of consistency (not shown).

The magnitude of the precipitation mass sink contribution to the PV tendency is proportional to the product of the PV and the precipitation mass flux divergence, suggesting that the effect is most pronounced in regions characterized by strong removal of water vapor and cloud material (condensation, deposition, riming, aggregation, autoconversion, etc.) and large preexisting PV. In computing (9), we utilized the following MM5 output variables: rainwater density $\left(\rho_{\mathrm{ra}}\right)$, snow $\left(\rho_{\mathrm{sn}}\right)$, and graupel $\left(\rho_{\mathrm{gr}}\right)$. The terminal fall speeds were obtained from the mass-weighted equations for rain, snow, and graupel ( $W_{\mathrm{ra}}, W_{\mathrm{sn}}$, and $\left.W_{\mathrm{gr}}\right)$ provided by Lin et al. (1983), which are consistent with the grid-scale microphysics scheme used in the MM5 simulation presented herein. The MM5 variables were initially output on 38 sigma levels and then were interpolated onto 65 constant height surfaces from 0 to $16 \mathrm{~km}$ at $250-\mathrm{m}$ increments. These height-coordinate data were then used to calculate the diabatic and mass sink PV tendency terms; calculations were not performed at the two upper and lowermost levels in order to maintain centered differencing in the vertical. The output from the PV budget com-

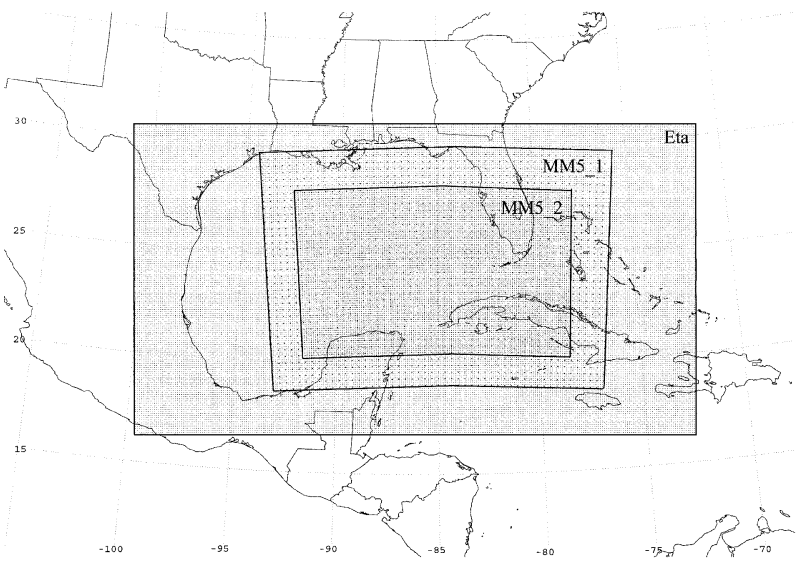

FIG. 2. Model domain configurations for MM5 and Eta Model simulations; MM5 domain 1 has 36-km grid spacing, MM5 domain 2 has $12-\mathrm{km}$ grid spacing, and the Eta domain has $15-\mathrm{km}$ grid spacing.

putations for the case of Hurricane Lili will be presented in section $4 \mathrm{c}$.

\section{Model experiments}

Experiments from two different primitive equation NWP models will be presented here. For computation of the mass and PV budgets, a simulation of Hurricane Lili from MM5 is utilized. Then, an alternate examination of the mass sink effect is made using experiments from the workstation Eta Model (Mesinger 1984), available from NCEP. The rationale for using two different models stems from the fact that MM5 gives a more realistic representation of the storm, including circulation strength and precipitation amounts. However, the mass sink effect is more straightforward to incorporate into a hydrostatic model such as the Eta, and this model was also found to produce a realistic tropical cyclone structure, albeit weaker than that from MM5.

\section{a. MM5 simulations}

The MM5 includes numerous options for grid nesting and physical parameterization and has been previously shown to produce successful hurricane simulations (e.g., Zhang et al. 2000, 2001, 2002; Braun and Tao 2000; Braun 2002; Davis and Bosart 2001, 2002). The precipitation field generated from MM5 simulations of Hurricane Lili is used to quantify the importance of the mass sink relative to other processes. Our objective is not to obtain a perfect MM5 simulation of Hurricane Lili, but rather to obtain a sufficiently realistic simulation to use as a physically consistent dataset with which to compute terms in the pressure- and PV-tendency equations.

Results are presented from a simulation with the oneway-nested domain depicted in Fig. 2; the grid spacing was $36 \mathrm{~km}$ for the outer domain and $12 \mathrm{~km}$ on the inner domain. The simulation was run with 38 vertical levels, 
the Betts-Miller cumulus parameterization (on both domains), the medium-range forecast (MRF) PBL scheme, the Goddard cloud microphysics scheme, and the cloudradiation scheme. All MM5 output presented below is from the $12-\mathrm{km}$ grid.

\section{b. Eta simulations}

Modifications necessary to incorporate the precipitation mass sink effect into the Eta Model were provided by F. Mesinger and D. Jovic of NCEP. The Eta-coordinate continuity equation [Mesinger et al. 1988, their Eq. (2.5)] is

$$
\frac{\partial}{\partial \eta}\left(\frac{\partial p}{\partial t}\right)+\nabla \cdot\left(\mathbf{v} \frac{\partial p}{\partial \eta}\right)+\frac{\partial}{\partial \eta}\left(\dot{\eta} \frac{\partial p}{\partial \eta}\right)=0,
$$

where $\eta \equiv\left(p-p_{T}\right) /\left(p_{S}-p_{T}\right) \eta_{S}$, and $\eta_{S}=\left[p_{\text {ref }}\left(\mathrm{z}_{S}\right)-\right.$ $\left.p_{T}\right] /\left[p_{\text {ref }}(0)-p_{T}\right]$ (Mesinger 1984; Mesinger et al. 1988). The modified form of (13), including sources and sinks due to water vapor phase changes, is given by

$\frac{\partial}{\partial \eta}\left(\frac{\partial p}{\partial t}\right)+\nabla \cdot\left(\mathbf{v} \frac{\partial p}{\partial \eta}\right)+\frac{\partial}{\partial \eta}\left(\dot{\eta} \frac{\partial p}{\partial \eta}\right)-\frac{d q}{d t} \frac{\partial p}{\partial \eta}=0$.

A modified surface-pressure tendency equation, obtained from integration of (14) from the top to the bottom of the model atmosphere and a modified computation of the kinematic omega are also included in the mass sink version of the model. Because condensate is not immediately removed to the surface, the pressure increase due to water loading is also included.

Numerous simulations of Lili were obtained using the workstation version of the Eta Model. The model simulations presented here utilized the Kain-Fritsch cumulus parameterization scheme because runs with this scheme resulted in the formation of a stronger (and more realistic) tropical cyclone relative to simulations using the Betts-Miller-Janjic scheme, which is used in the operational version of the Eta Model. In general, the Eta produced a weaker storm than did the MM5, even though both sets of simulations were initialized from the same GDAS analysis of 0000 UTC 1 October 2002. The Eta simulations presented here were run on a 15$\mathrm{km}$ grid (Fig. 2) with 60 vertical levels. The control (CTRL) and experimental (MSNK) runs were identical in every respect except for the modifications outlined above.

\section{c. Initial conditions}

Initial fields from the NCEP Eta Data Assimilation System (EDAS) proved inadequate for model initialization due to a poor analyzed representation of Lili. Although coarser in resolution, an advantage of the NCEP Global Forecast System (GFS) data assimilation system (GDAS) is that the operational system in place at the time of Lili included an adjustment of location of the incipient storm to the correct position using in- formation provided by the Tropical Prediction Center (TPC). In 2002, the GDAS system did not insert an idealized vortex into the initial condition analysis, but there was an initial disturbance of sufficient amplitude to allow vigorous development in the MM5 simulation for this case. For detailed discussions on this point, see, for example, Kurihara et al. (1993, 1995), Mesinger (1998), Liu et al. (2000), and Pu and Braun (2001). A GDAS grid with approximately $1^{\circ}(95 \mathrm{~km})$ grid spacing available at 6-h intervals provided initial and lateral boundary conditions for the Eta (MM5) simulations.

The MM5 simulation presented here was initialized from the GDAS analysis at 0000 UTC 1 October and run through 1200 UTC 2 October in order to capture the main period of intensification. As expected, the coarse resolution of the initial conditions did not capture the full intensity of the observed system, so the model storm began with a central pressure that was approximately $19 \mathrm{hPa}$ greater than that observed in Lili at the initial time. Based on results from the MM5 simulation, all Eta simulations were initialized using GDAS data from 0000 UTC 1 October 2002 and run out to $48 \mathrm{~h}$ (0000 UTC 3 October).

\section{Application to Hurricane Lili (2002)}

\section{a. Storm overview and MM5 simulation}

Hurricane Lili (30 September-3 October 2002) was selected for analysis because it developed sufficiently close to populated land areas to increase confidence in the quality of the analyzed initial dataset (due in part to aircraft reconnaissance data). On 16 September 2002, the tropical wave that would eventually become Hurricane Lili moved westward over the Atlantic Ocean from the west coast of Africa (Lawrence 2003). Continuing westward and then west-northwestward, Lili attained hurricane status upon reaching the Little Cayman Islands on 30 September (Fig. 3). By 0000 UTC 1 October, Lili was centered near $20.5^{\circ} \mathrm{N}, 81.1^{\circ} \mathrm{W}$, with an estimated central pressure of $978 \mathrm{hPa}$. After a pause in intensification while Lili moved over the southwestern tip of the Isle of Youth and then over western mainland Cuba on 1 October, intensification had resumed by 0000 UTC 2 October. At 0000 UTC 2 October the central pressure was estimated at $967 \mathrm{hPa}$, falling to $954 \mathrm{hPa}$ by 1200 UTC 2 October, with estimated maximum winds of $110 \mathrm{kt}$. Strengthening continued until approximately 2000 UTC 2 October, at which time the central pressure had fallen to $938 \mathrm{hPa}$ and peak winds had reached $125 \mathrm{kt}$. Lili underwent unexpected weakening early on 3 October, as the central pressure increased to $963 \mathrm{hPa}$ and maximum sustained winds decreased to 80 kt prior to landfall at 1300 UTC 3 October near Intracoastal City, Louisiana.

Lili's central pressure in the MM5 simulation decreased from $987 \mathrm{hPa}$ at $12 \mathrm{~h}$ (1200 UTC 1 October) to $966 \mathrm{hPa}$ by $36 \mathrm{~h}$ (1200 UTC 2 October), with $10-\mathrm{m}$ 


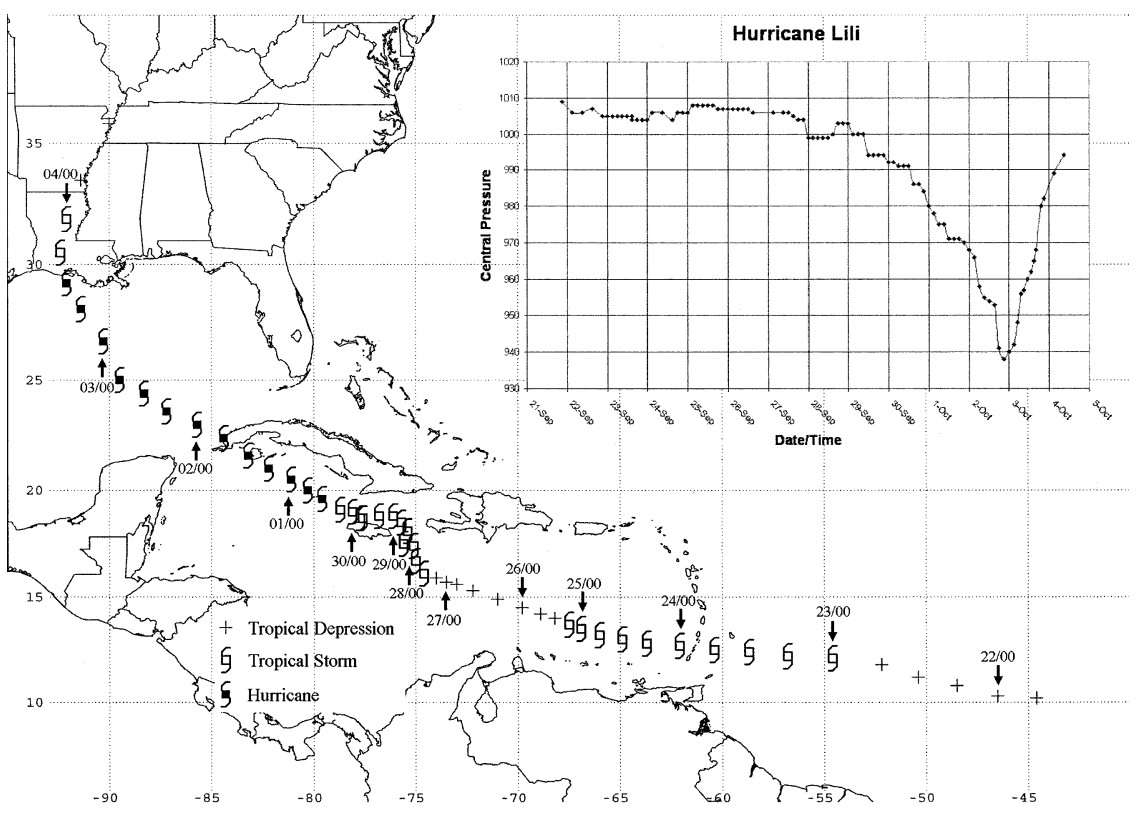

FIG. 3. Hurricane Lili track with date/hour provided. Inset depicts central pressure of Lili. Symbols denote storm classification as indicated in the legend. The track and central pressure data were obtained from the Tropical Prediction Center (http://www.nhc.noaa.gov/2002lili.shtml).

wind speeds exceeding $70 \mathrm{kt}$ at that time (Fig. 4). Although the model storm deepened considerably during the simulation, the model central pressure remained 10$20 \mathrm{hPa}$ higher than observed. This discrepancy is likely due to a combination of insufficient initial storm intensity, resolution, and limitations in model physics. This overestimate of storm central pressure is perhaps in part attributable to the choice of the MRF PBL scheme, which produces an overly deep boundary layer and allows for drying there, as shown by Braun and Tao (2000). Additional simulations with alternative PBL schemes were found to produce greater deepening (not shown). Despite the differences in central pressure from observations, the MM5 simulation produced a realistic storm structure, including a warm core vortex, an "eye" in the precipitation pattern (Fig. 5), and a deepening rate similar to that observed.

The mass and PV budget analyses will focus on the period from 0600 UTC to 1200 UTC 2 October, when Lili was deepening steadily. Peak hourly precipitation rates in the MM5 simulation exceeded $50 \mathrm{~mm}$ in the eyewall (Fig. 5). In order to provide a qualitative comparison between the model precipitation field and observational data, we obtained imagery from the Tropical Rainfall Measurement Mission (TRMM) from the Naval Research Laboratory Web page. The TRMM image from 1254 UTC 2 October indicates rain rates in excess of $25 \mathrm{~mm} \mathrm{~h}^{-1}$ (Fig. 6). It is superimposed with the Geostationary Operational Environmental Satellite-8 (GOES-8) visible image for 1315 UTC 2 October. The TRMM image from 0622 UTC indicated hourly rain rates greater than $30 \mathrm{~mm} \mathrm{~h}^{-1}$ (not shown).

\section{b. Mass budget analysis}

As an initial quantitative test of our hypothesis that the precipitation mass sink is not negligible in tropical cyclones, the terms in (6) were computed for a cylindrical region of $100-\mathrm{km}$ radius centered on Lili. The net surface-pressure tendency is determined by the small difference between strong mass convergence in the lower troposphere, mass divergence aloft, and mass loss due to precipitation. Owing to the fact that the MM5 model does not account for the mass sink in the pressure-tendency equation, we can assume that the left side of (6) from the model would very nearly balance the first right-hand term. Although the evaporation rate within $100 \mathrm{~km}$ of the storm center is probably not negligible, we can safely assume that the net precipitation within this radius is much greater than the evaporation there (Palmén and Riehl 1957). If the area-averaged pressure tendency due to the precipitation mass sink term is small relative to the left-hand term in (6), we can dismiss the importance of this process at least in a linear sense (aside from possible feedbacks involving moisture convergence and vorticity). If the mass removed due to precipitation were comparable in magnitude to the left-hand side, it would indicate that the precipitation mass sink is a potentially important component of the tropical cyclone mass budget.

The average model pressure over a circle of $100-\mathrm{km}$ radius centered on the storm was computed for hours 30 through 35 in the MM5 simulation. Storm motion and location were determined from hourly plots of model surface pressure, allowing for objective interpolation 

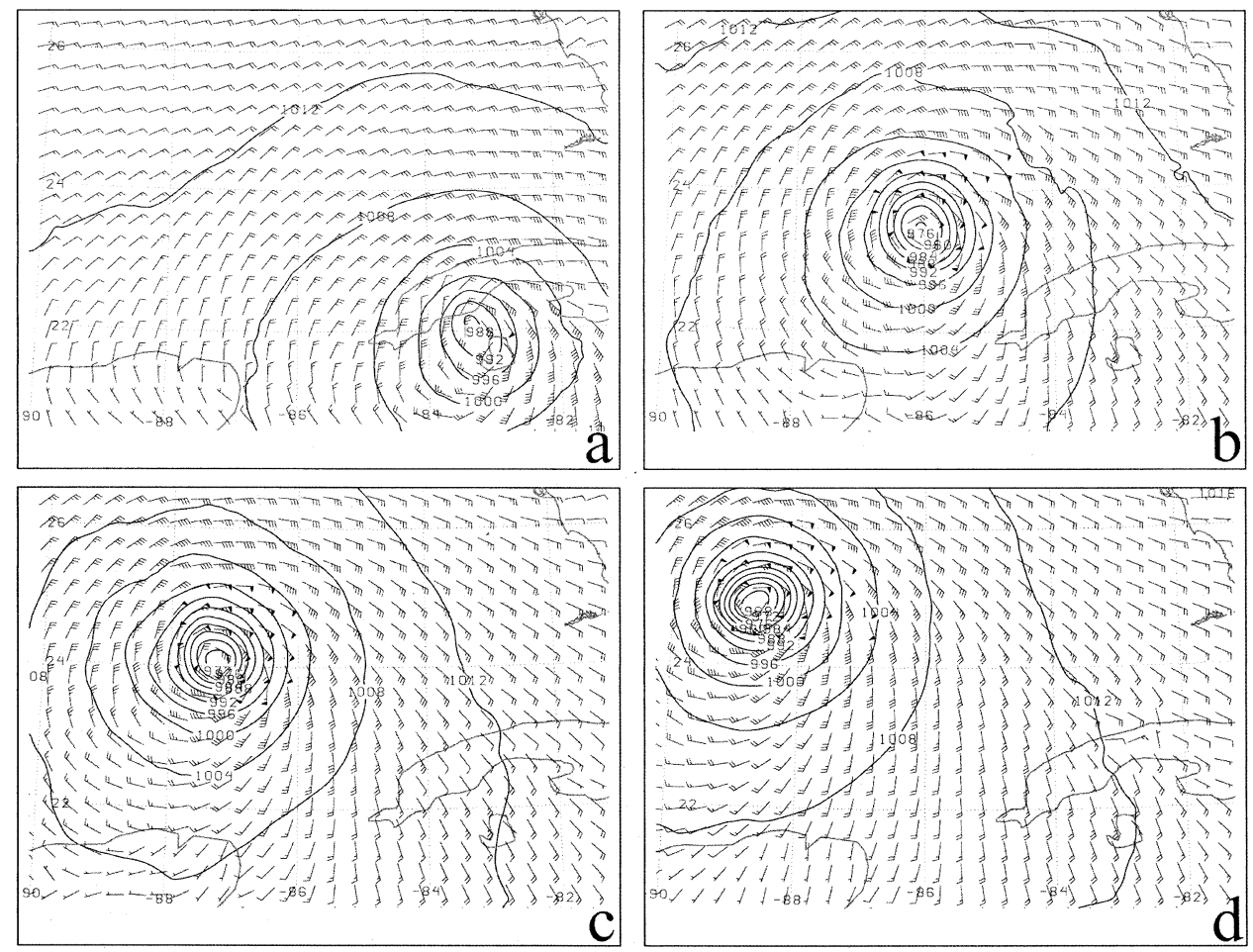

FIG. 4. Sequence of sea level pressure (solid, contour interval $4 \mathrm{hPa}$ ) and 10-m wind (standard plotting convention) from MM5 simulation: (a) 12-h simulation valid at 1200 UTC 1 Oct, (b) 24-h simulation valid at 0000 UTC 2 Oct, (c) 30-h simulation valid at 0600 UTC 2 Oct, and (d) 36-h simulation valid at 1200 UTC 2 Oct.

to obtain the minimum value between grid points. Between 230 and 233 model grid points were involved in each calculation. The average pressure in the storm domain decreased steadily through this period, yielding an average hourly pressure tendency of approximately $-0.5 \mathrm{hPa}$ and a total $5-\mathrm{h}$ pressure fall of $-2.3 \mathrm{hPa}$ (Table 1). The average hourly pressure-equivalent mass loss due to precipitation within the cylinder was $-1.5 \mathrm{hPa}$, yielding a total of $-7.3 \mathrm{hPa}$. These results demonstrate that the amount of atmospheric mass removed via precipitation exceeded that needed to explain the model sea level pressure decrease. Although the Eta simulations presented here produced lighter precipitation relative to the MM5 simulation (by roughly a factor of 3 ), the mass loss is still of the same order as that needed to explain the observed pressure decrease. ${ }^{5}$ Thus, we cannot dismiss the precipitation mass sink as negligible near the center of the strengthening tropical storm.

\section{c. Potential vorticity equation results}

Several earlier studies have examined the evolution of PV during the development of tropical cyclones (e.g.,

\footnotetext{
${ }^{5}$ The TPC central pressure estimates indicate an average deepening rate of approximately $1.1 \mathrm{hPa} \mathrm{h}^{-1}$ for the 24-h period from $0000 \mathrm{UTC}$ 2 October to 0000 UTC 3 October, and so the mass budget result would hold even if the MM5 storm had deepened at the same rate as the observed storm.
}

Schubert and Alworth 1987; Möller and Smith 1994; Davis and Bosart 2001, 2002), yet the authors are unaware of previous studies that specifically quantify the contribution of the precipitation mass sink to the local PV tendency. Figure 7 presents PV and winds for the $1-3-\mathrm{km}$ and $9-10-\mathrm{km}$ layers for hour 34 of the MM5 simulation. In this simulation, Lili's cyclonic PV tower extended to near the 250-hPa level, consistent with sustained cyclonic flow even at that level (Figs. 7b, 8). A cross section through the PV tower reveals extremely strong latent heat release (exceeding $20 \mathrm{~K} \mathrm{~h}^{-1}$ over much of the depth of the troposphere) along the outer periphery of the tower, surrounding a warm core region of small heating (Fig. 8a). In the center of the storm, the freezing level is higher in altitude relative to ambient regions. Negative diabatic temperature tendencies are found outside the eyewall heating maximum and near the surface beneath the eyewall heating maximum (Fig. $8 \mathrm{a})$. There is a suggestion of the "stadium effect" as the heating maxima lean outward from the storm center on either side of the eye. The diabatic PV tendency can be determined from the projection of the latent heating gradient onto the absolute vorticity vector as shown by (11). Given the very large magnitude of the heating gradient in the vicinity of the eyewall, one would expect a rather noisy PV-tendency field. The resulting diabatic PV flux vectors (due to latent heat release) and the corresponding local PV tendency are shown in Fig. 8b. A 

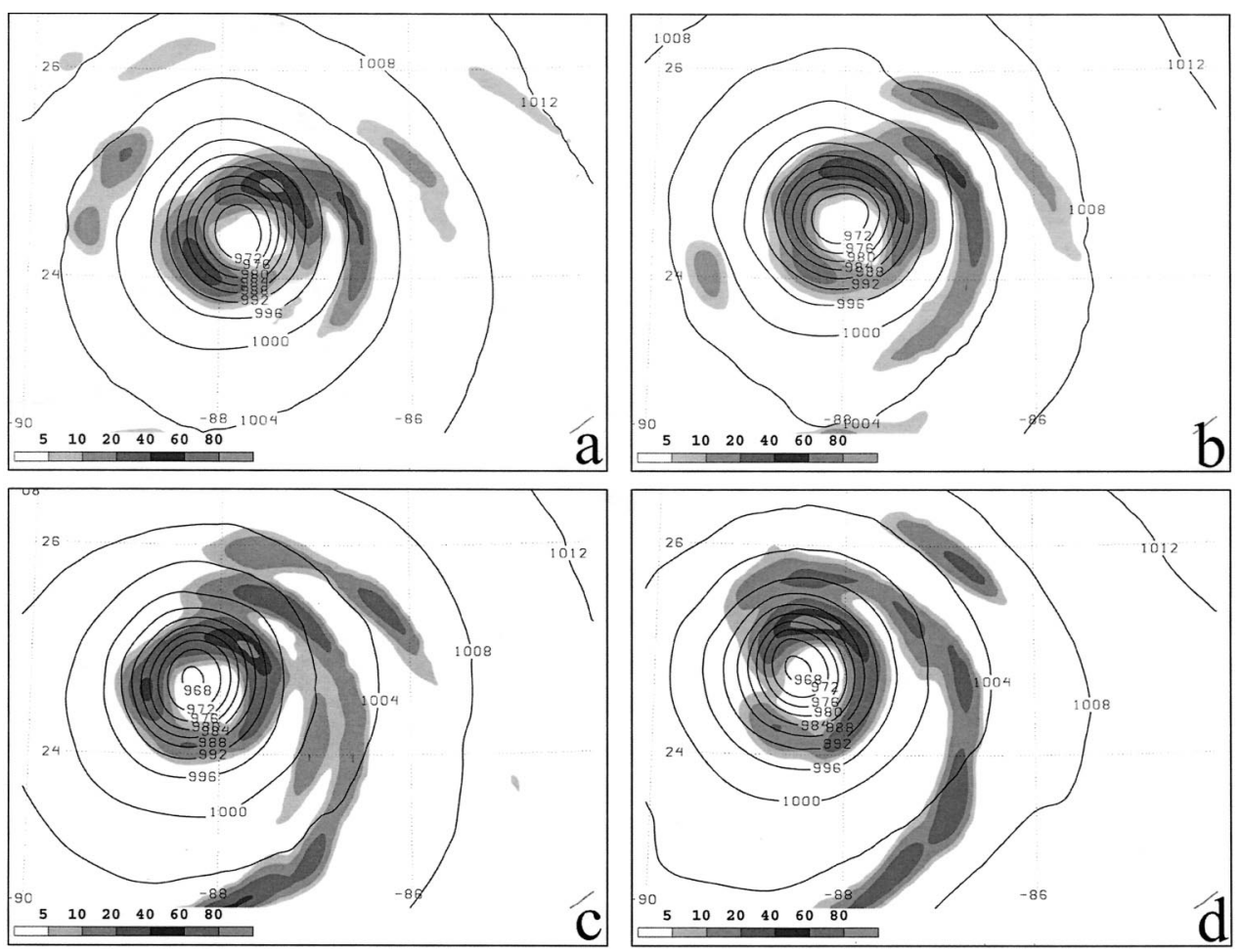

FIG. 5. Sequence of sea level pressure and hourly precipitation (mm, shaded as in legend): (a) 32-h simulation valid at $0800 \mathrm{UTC}$, (b) 33-h simulation valid at $0900 \mathrm{UTC}$, (c) 34-h simulation valid at $1000 \mathrm{UTC}$, and (d) 35-h simulation valid at 1100 UTC 2 Oct.

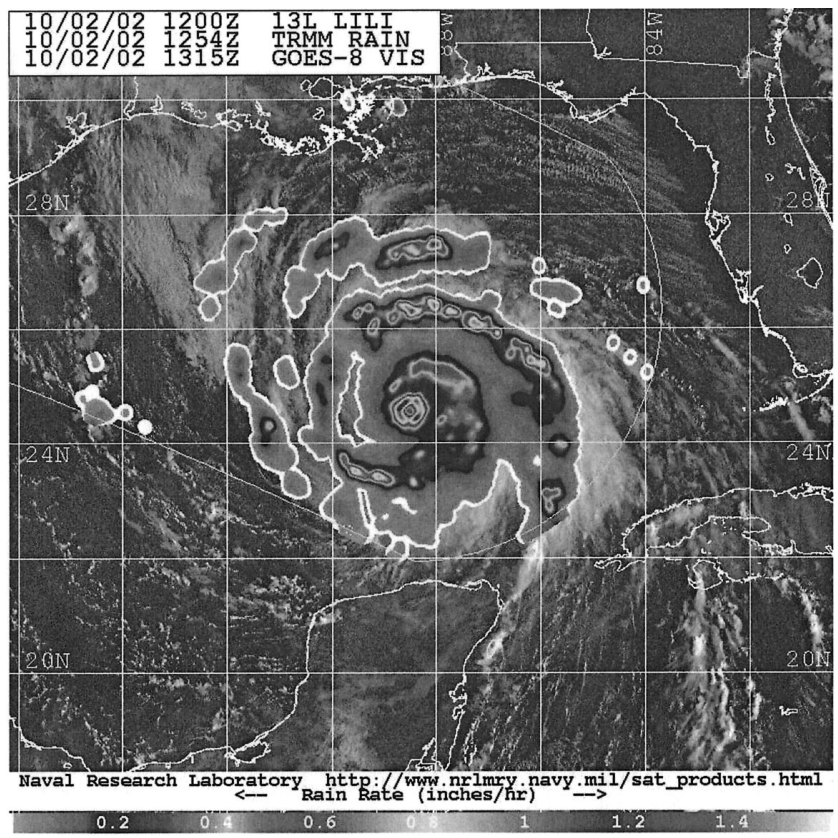

FIG. 6. Rainfall rate $\left(\mathrm{h}^{-1}\right)$ from TRMM satellite (shaded as indicated in legend) for 1254 UTC 2 Oct 2002, superimposed with GOES-8 visible image for 1315 UTC 2 Oct 2002. Image courtesy of Naval Research Laboratory, from http://www.nrlmry.navy.mil/sat_products.html. 
TABLE 1. Mass budget based on MM5 simulation for cylinder of 100-km radius centered on Hurricane Lili. Between 230 and 233 model grid points were used in each calculation.

\begin{tabular}{cccc}
\hline \hline & $\begin{array}{c}\text { Average pressure } \\
(100-\mathrm{km} \text { cylinder } \\
\text { centered on storm) }\end{array}$ & $\begin{array}{c}\text { Hourly pressure change } \\
\text { (following storm center) }\end{array}$ & $\begin{array}{c}\text { Pressure equivalent of } \\
\text { precipitation mass sink }\end{array}$ \\
\hline 30 & 988.54 & - & -1.56 \\
31 & 987.76 & -0.78 & -1.46 \\
32 & 987.53 & -0.54 & -1.42 \\
33 & 986.99 & -0.28 & -1.37 \\
34 & 986.71 & -0.46 & -1.44 \\
35 & 986.25 & $-2.29 \mathrm{hPa} /-0.46 \mathrm{hPa} \mathrm{h}^{-1}$ & $-7.25 \mathrm{hPa} /-1.45 \mathrm{hPa} \mathrm{h}-1$ \\
Total/avg & &
\end{tabular}

strong downward PV flux is found throughout the eyewall region, while the diabatic PV tendency exhibits very large values, exceeding $25 \mathrm{PVU} \mathrm{h}^{-1}$ (where 1 PVU $\left.=1.0 \times 10^{-6} \mathrm{~m}^{2} \mathrm{~s}^{-1} \mathrm{~K} \mathrm{~kg}^{-1}\right)$. In a Lagrangian sense, air parcels rising in the eyewall would experience alternating periods of strong PV growth and decay.

The instantaneous PV tendency due to the precipitation mass sink (12) is almost entirely positive and is maximized in the vicinity of the melting layer (Fig. 9). The mass sink term is nearly two orders of magnitude smaller than the diabatic term (11). However, in contrast to the noisy signal produced from the diabatic term (Fig. $8 b$ ), the mass sink term provided a well-organized contribution on the order of $10 \mathrm{PVU}_{\text {day }^{-1}}$. A complete explanation for the PV-tendency pattern shown in Fig. 9 will require additional analysis, including an account of hydrometeor trajectories. We speculate that the increased fall velocity below the freezing level leads to increased vapor and cloud water removal efficiency, contributing to precipitation mass flux divergence there. ${ }^{6}$ In a Lagrangian sense, rising air parcels gain PV due to the mass sink term until reaching the freezing level, so one would expect the maximum cyclonic PV accumulation due to this process to reside above this altitude.

Volume-integrated PV tendencies were computed for the same cylindrical volume as for the mass budget (not shown). The diabatic PV tendency was much larger than that due to the precipitation mass sink, but the former exhibited strong spatial cancellation so that the contribution of the latter was not negligible in a volumeaveraged sense. Due to large noise in the diabatic PV computation, a more accurate approach would be to code the PV budget equation directly into a model in order to accumulate PV due to different processes in a manner similar to that presented by Stoelinga (1996). Another approach, presented in the following section, will be to present PV difference fields between the control and mass sink simulations from the Eta Model.

\footnotetext{
${ }^{6}$ Above the freezing level, it appears that the relatively small fall velocity of snow $\left(\sim 1 \mathrm{~m} \mathrm{~s}^{-1}\right)$ in conjunction with strong updraft velocities $\left(>1 \mathrm{~m} \mathrm{~s}^{-1}\right)$ leads to lofting and lateral centrifuging of hydrometeor material.
}

\section{d. Eta Model sensitivity experiments}

As discussed in section $3 \mathrm{~b}$, a rigorous test of our hypothesis is provided by comparison of two numerical model simulations, one of which is based on a modified version of the model incorporating the mass sink effect. The control (CTRL) and experimental (MSNK) simulations are identical in every respect except for the modifications outlined in section 3b. At 24 h, valid 0000 UTC 2 October, the Eta control simulation (CTRL) produced modest deepening of the storm relative to the corresponding MM5 simulation (Figs. 10a and 4b); however, the Eta was able to reproduce a realistic tropical cyclone structure, including an eyelike feature in the precipitation field. The MSNK simulation had produced an additional deepening of 3-4 $\mathrm{hPa}$ beyond CTRL (Figs. 10a,b) by this time, with the strongest pressure differences centered near the storm (Fig. 10d). Throughout the integration, the MSNK simulation tended to produce a precipitation field that more completely encircled the cyclone center, as is evident from comparison of Figs. 10a,b. The difference field for 3-hourly precipitation, shown in Fig. 10c, demonstrates that the MSNK run produced generally heavier precipitation, especially northeast of the storm, where differences exceeded $15 \mathrm{~mm}(3 \mathrm{~h})^{-1}$. Differences in the model $10-\mathrm{m}$ wind field reveals that the MSNK simulation was characterized by stronger winds of generally 2-7 kt at this time (Fig. 11), as would be expected from the pressure differences. The vector difference, presented in Fig. 11d, indicates a convergent, cyclonic pattern, consistent with the stronger storm in MSNK relative to CTRL.

The 30-h Eta simulations (Fig. 12), valid 0600 UTC 2 October, are directly comparable to the MM5 simulation shown in Fig. 4c. Again, the Eta failed to produce the tight inner core seen in the MM5 simulation despite similar horizontal grid spacing, but nevertheless depicted a strengthening tropical cyclone with deepening rates in excess of $12 \mathrm{hPa}$ in $30 \mathrm{~h}$. The MSNK run continues to depict a deeper storm with a central pressure more than $4 \mathrm{hPa}$ lower than that in CTRL. Given the modest deepening produced by the Eta, this difference represents approximately a $30 \%$ increase in deepening from CTRL. Examination of Fig. 12 indicates that the pressure differences are spatially uniform and are not 

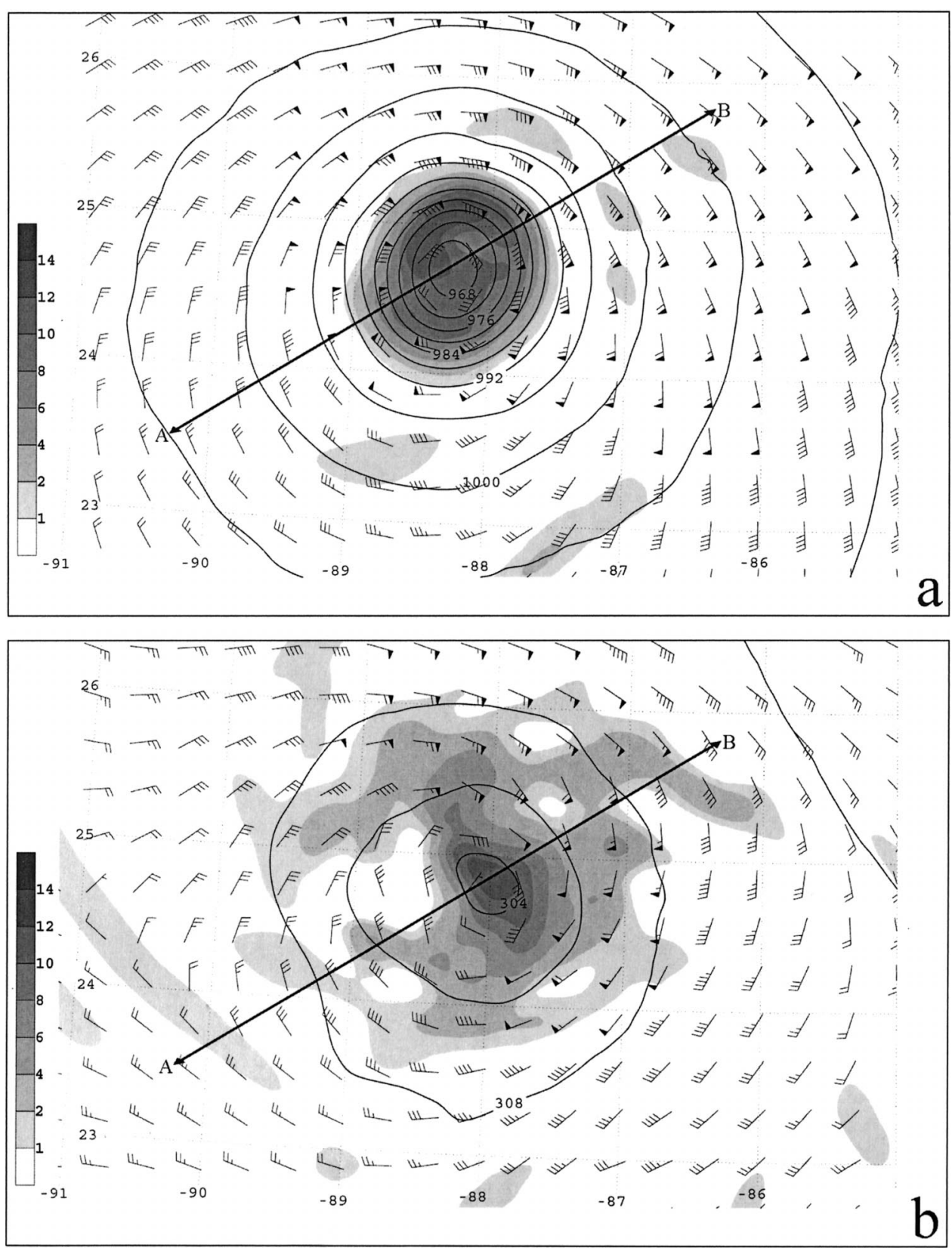

FIG. 7. Potential vorticity summary for 1000 UTC 2 Oct: (a) 1-3-km potential vorticity (PVU, shaded as in legend), layer-average wind (every third point plotted, standard convention), and sea level pressure (solid contours, 4-hPa interval); (b) as in (a) except for 9-10-km layer and pressure at $9.5-\mathrm{km}$ level (contour interval $2 \mathrm{hPa}$ ). Bold line A-B indicates location of cross section shown in Figs. 8 and 9.

due to slight differences in the storm location between the two simulations. Wind speed differences exhibit a similar pattern to those at $24 \mathrm{~h}$, with the largest differences $(>10 \mathrm{kt})$ to the west and south of the storm center (Fig. 13).

The differences between CTRL and MSNK do not continue to amplify with time, perhaps due to the increasingly close proximity of the northwestern lateral boundary, which is identical in each run. By $48 \mathrm{~h}$, the
MSNK run remained 3-4 hPa deeper and continued to exhibit a more coherent precipitation field encircling the storm center relative to CTRL. The spatial pattern of the pressure differences has become larger and more diffuse by this time. Large differences in 3-hourly precipitation had developed between the two simulations (Fig. 14c), with maximum differences exceeding 100 $\mathrm{mm}$ (not contoured) by this time. These large differences at this time are in part due to spatial offset of localized 

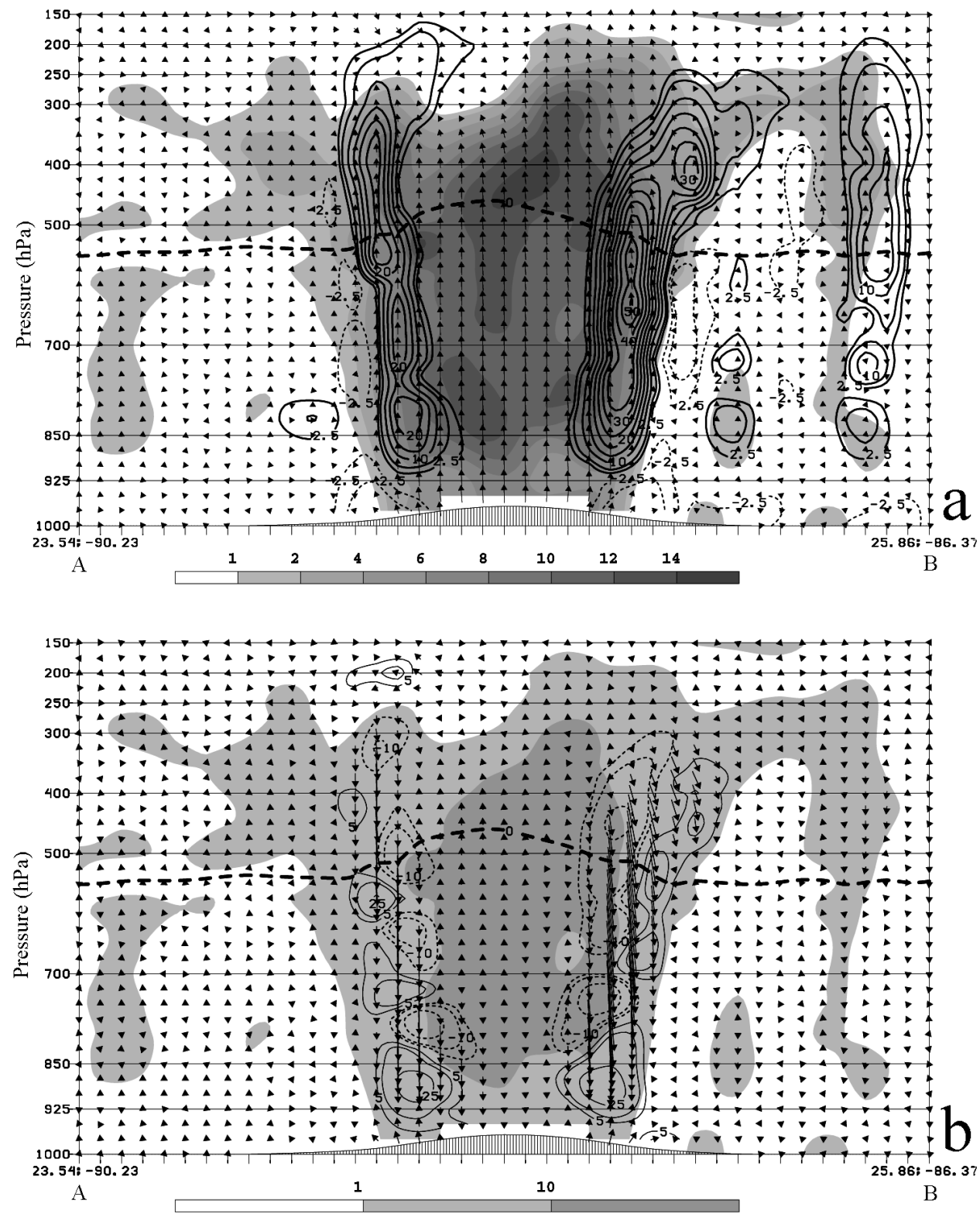

FIG. 8. Potential vorticity budget cross section A-B for 1000 UTC 2 Oct, depicting (a) PV (PVU, shaded as indicated in legend), latent heating rate (contour interval $5 \mathrm{~K} \mathrm{~h}^{-1}$, with $2.5 \mathrm{~K} \mathrm{~h}^{-1}$ contour added), absolute vorticity vector, and 273-K isotherm (thick dashed line); (b) as in (a) except with PV tendency due to latent heat release $\left(\mathrm{PVU} \mathrm{h}^{-1}\right.$, contoured) and diabatic PV flux vectors. Section orientation is shown in Fig. 7.

heavy precipitation features embedded in the eyewall. As was the case at 24 and $30 \mathrm{~h}$, the MSNK run continues to exhibit a more complete eyewall structure and generally heavier precipitation. The wind field differences have become more complex, as the MSNK storm has become more elongated in the zonal direction relative to the CTRL simulation (Fig. 15). The pattern of convergent flow in the vector difference wind field is still evident in Fig. 15d, consistent with overall heavier precipitation and a stronger storm.

Both Eta simulations produced a strong cyclonic PV tower that is comparable to that produced in the MM5 simulation. Figure 16 includes cross-sectional plots of Ertel PV through the CTRL and MSNK PV towers 24 $\mathrm{h}$ into the simulations, along with the PV difference field (Fig. 16c). The MSNK simulation exhibits a stronger PV tower in general, with greatest differences (exceeding 4 PVU) above the freezing level. This result is qualitatively consistent with the MM5 PV budget computations presented in Fig. 9, as rising parcels will have experienced a prolonged period of PV growth due to the mass sink process by the time they have reached this level. However, the difference field does not indicate a steady increase in PV growth below the freezing level. 


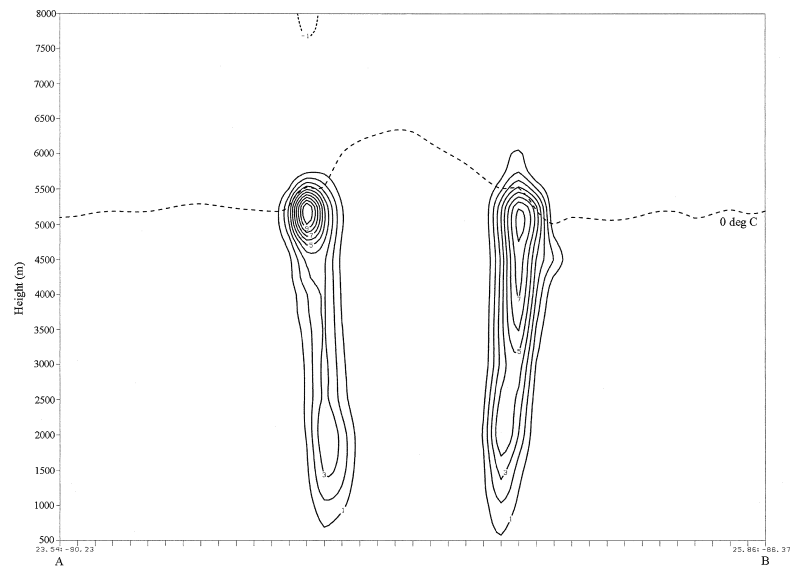

FIG. 9. Cross section A-B depicting potential vorticity tendency due to the precipitation mass sink (contour interval $1 \mathrm{PVU}^{\mathrm{Pay}}{ }^{-1}$ ) and the $273-\mathrm{K}$ isotherm (dashed line). Section orientation is shown in Fig. 7.

A second region of larger PV in the MSNK run is found near the surface, although a localized but strong region immediately below the melting level exhibits larger PV in CTRL.

The vertical structure of geopotential height and wind differences (Fig. 17) are consistent with the conceptual model presented in Fig. 1 and with the PV differences shown in Fig. 16, although it is perhaps surprising that the height differences are not larger above the freezing level where the PV differences are maximized. The MSNK run exhibited a stronger cyclonic circulation throughout the depth of the troposphere, with differences exceeding 10-15 kt both above and below the freezing level (Fig. 17a). The vector wind difference at the $700-\mathrm{hPa}$ level is shown in Fig. $17 \mathrm{~d}$ and indicates that a stronger cyclonic circulation was present in the MSNK run relative to CTRL. The divergence difference field was noisy, even after a nine-point horizontal smoother was applied, but it suggests stronger lowertropospheric convergence in the MSNK run (Fig. 17b), which is consistent with the $10-\mathrm{m}$ wind difference fields shown in Fig. 11.

The results of the Eta experiments are consistent with the initial hypothesis that the precipitation mass sink provides a nonnegligible contribution to the deepening of the storm and results in stronger winds, enhanced convergence, and heavier precipitation. While the differences between these simulations are not large in absolute terms, recall that neither Eta simulation deepened Lili to the extent that was observed. These results indicate that the mass sink process is not negligible, but also that this process does not play a dominant role in the storm dynamics.
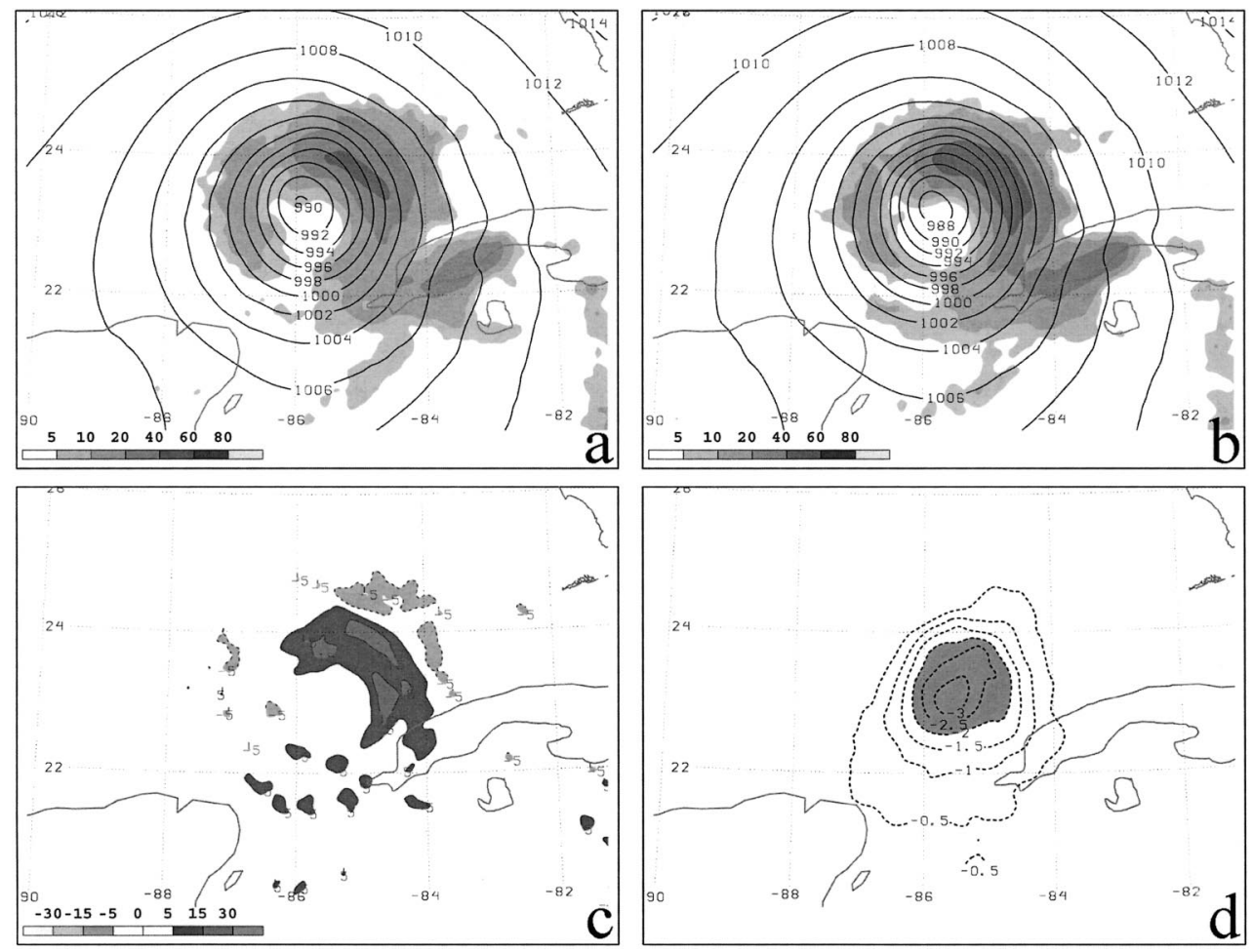

FIG. 10. Eta Model simulations valid at 0000 UTC 2 Oct 2002: (a) CTRL simulation sea level pressure (solid, contour interval $2 \mathrm{hPa}$ ) and 3-h precipitation total (mm, shaded as in legend), (b) as in (a) except for MSNK simulation; (c) difference (MSNK - CTRL) in precipitation (mm), contoured and shaded as in legend; and (d) difference (MSNK - CTRL) in sea level pressure, contour interval $0.5 \mathrm{hPa}$, shaded below $2 \mathrm{hPa}$. 

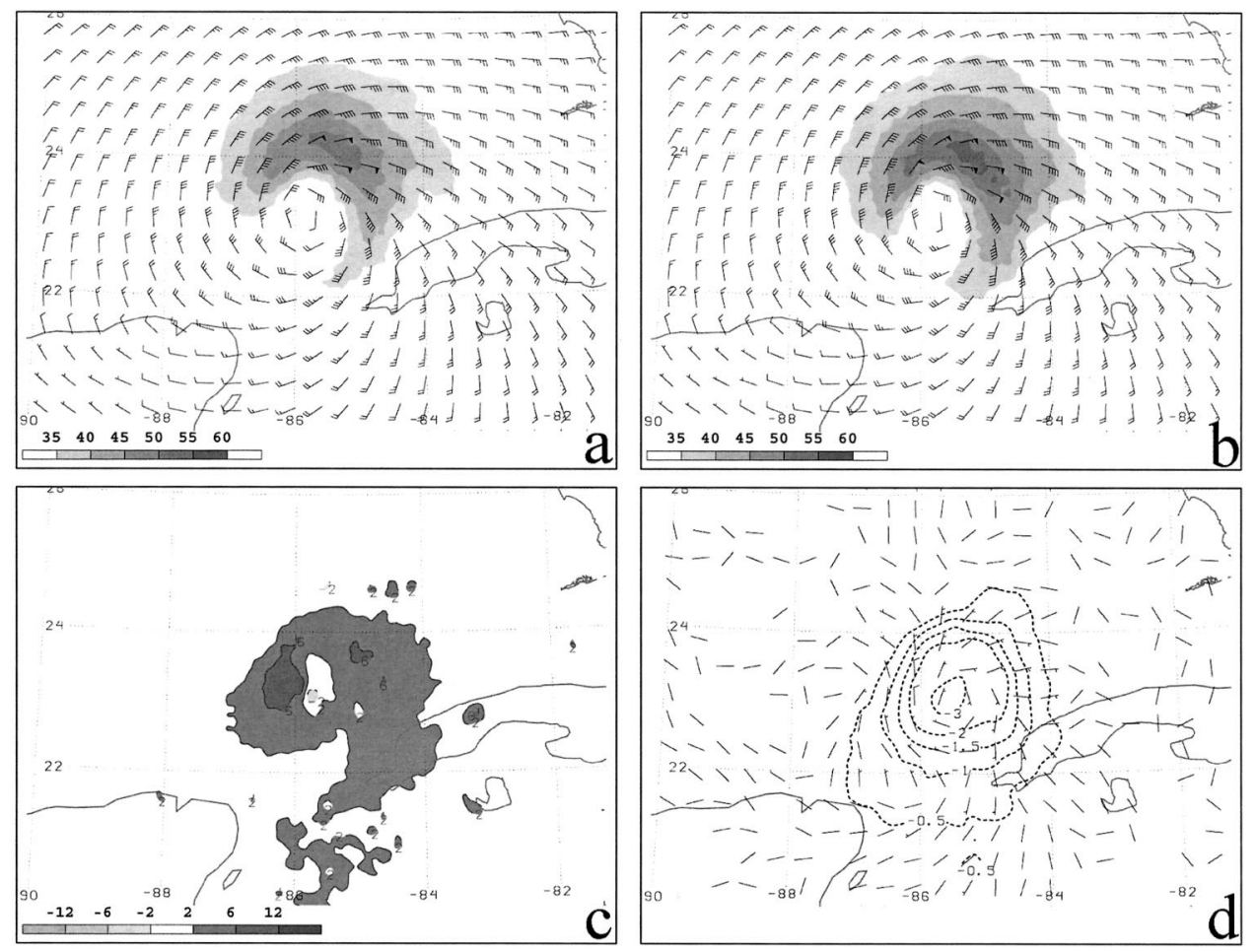

FIG. 11. Eta Model simulations of Hurricane Lili valid at 0000 UTC 2 Oct 2002: (a) CTRL simulation $10-\mathrm{m}$ wind speed (shaded as in legend) and wind barbs (standard plotting convention); (b) as in (a) except for MSNK simulation; (c) difference (MSNK - CTRL) in wind speed (kt), contoured and shaded as in legend; and (d) difference (MSNK - CTRL) in sea level pressure, contour interval $0.5 \mathrm{hPa}$, and vector difference in $10-\mathrm{m}$ wind (MSNK - CTRL)
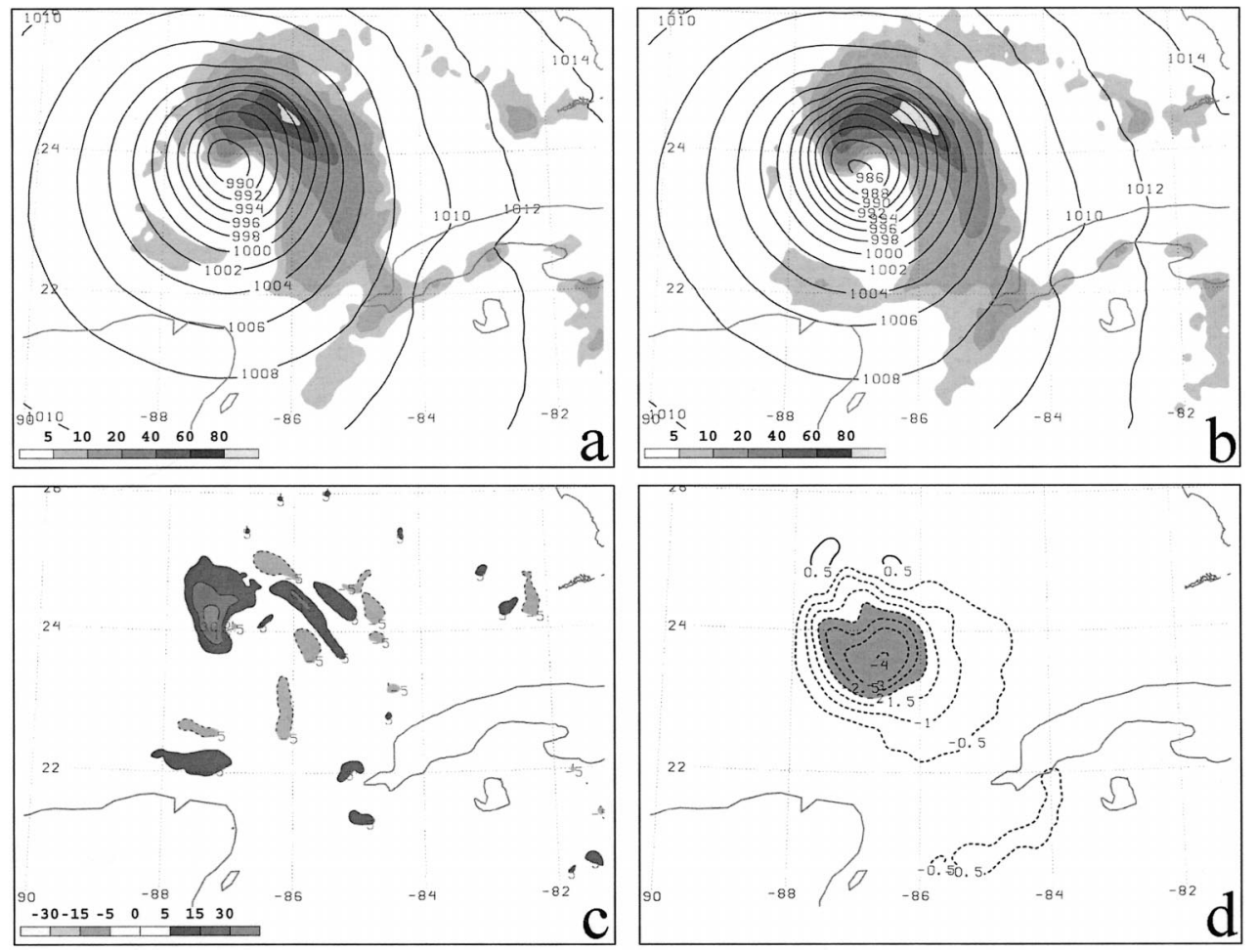

FIG. 12. As in Fig. 10 except for 30-h simulation valid at 0600 UTC 2 Oct. 

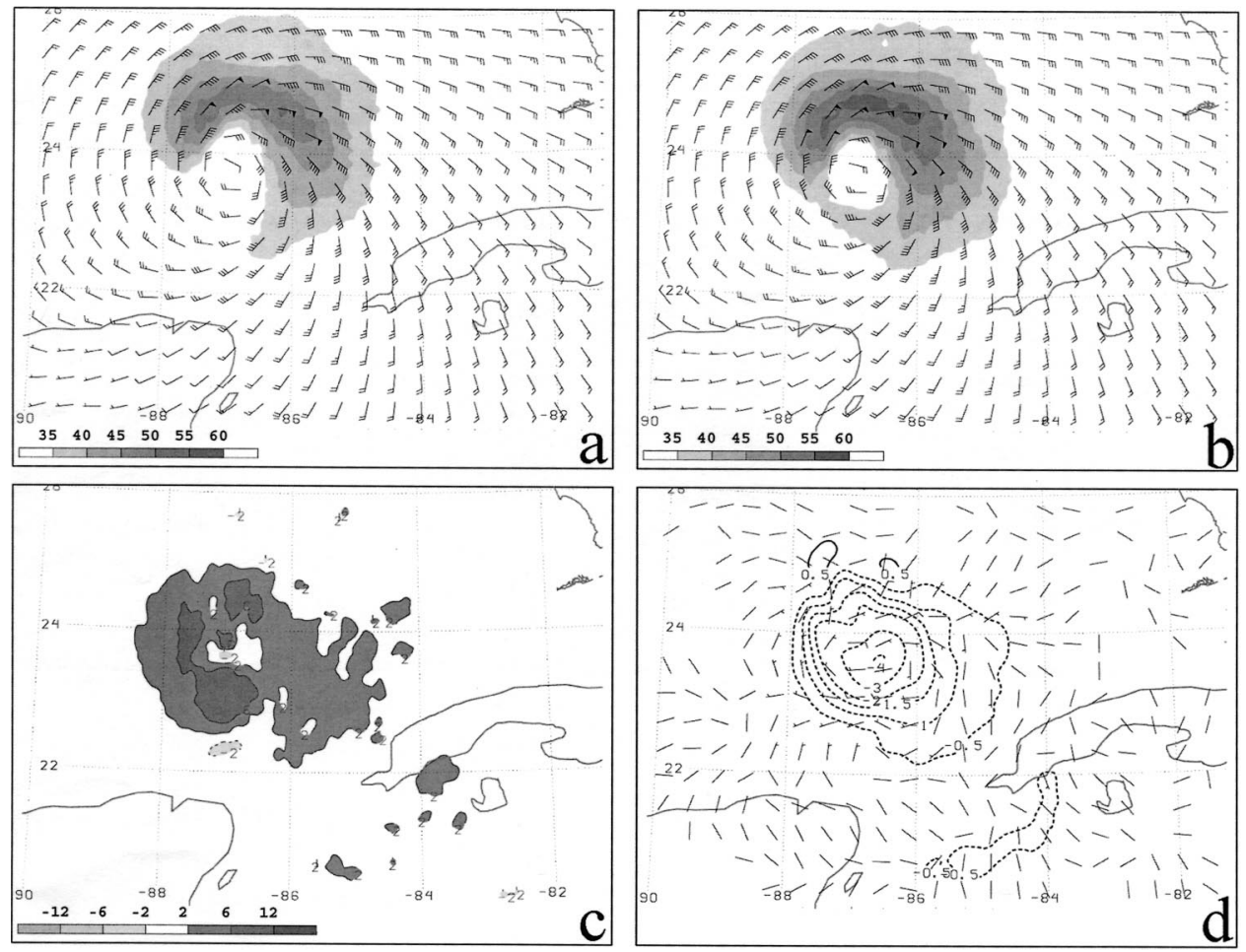

FIG. 13. As in Fig. 11 except for 30-h simulation valid at 0600 UTC 2 Oct.
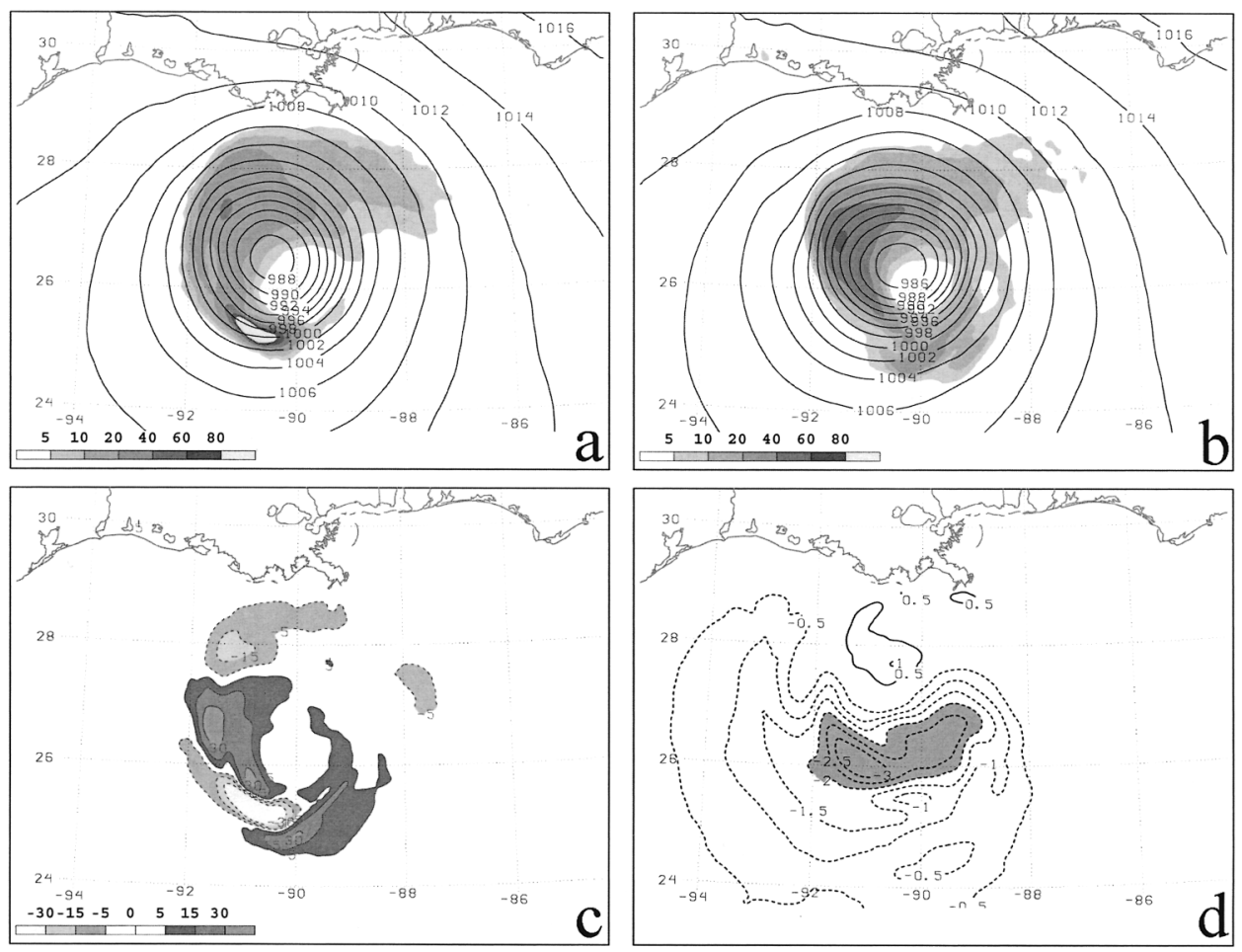

FIG. 14. As in Fig. 10 except for 48-h simulation valid 0000 UTC 3 Oct. 

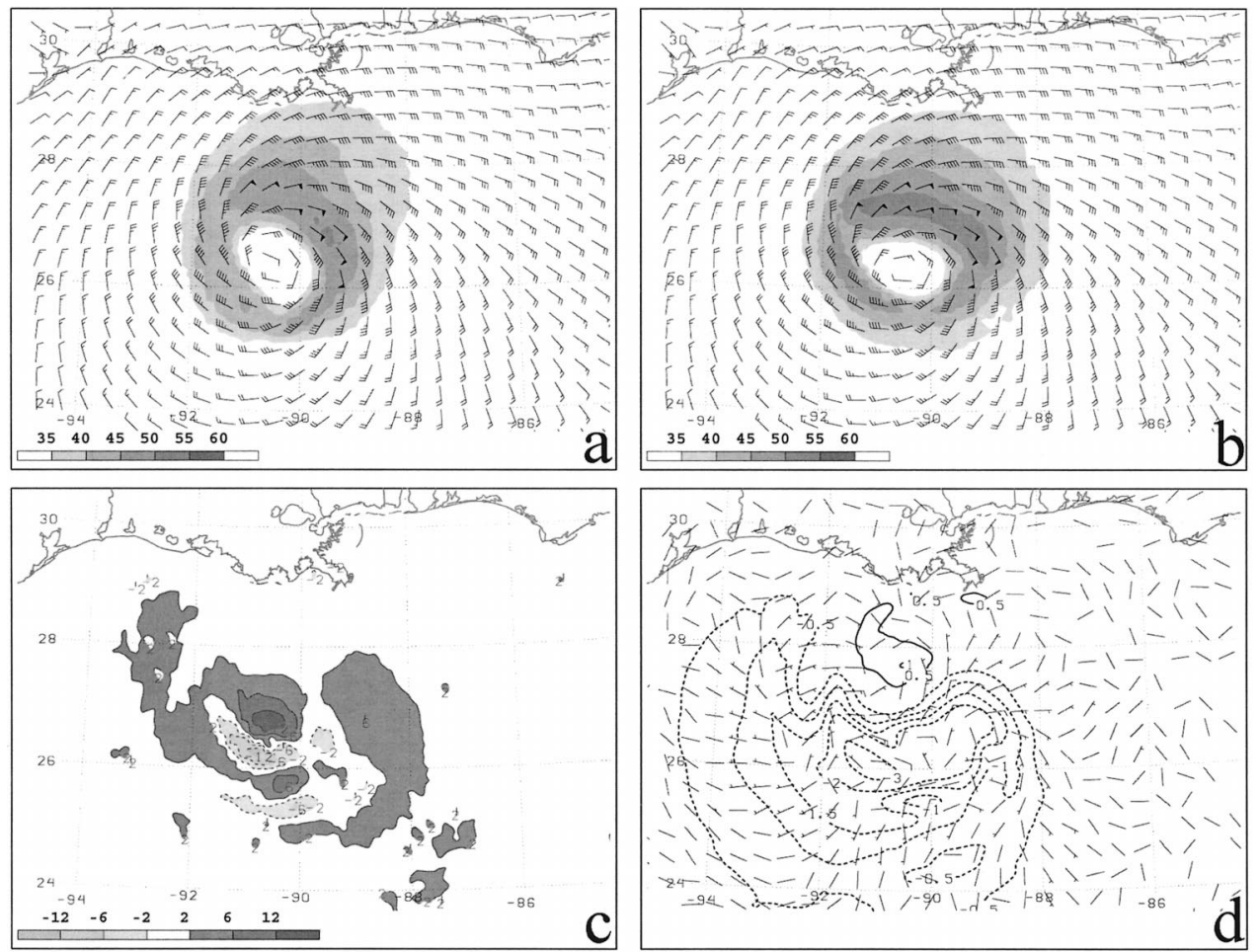

FIG. 15. As in Fig. 11 except for 48-h simulation valid at 0000 UTC 3 Oct.
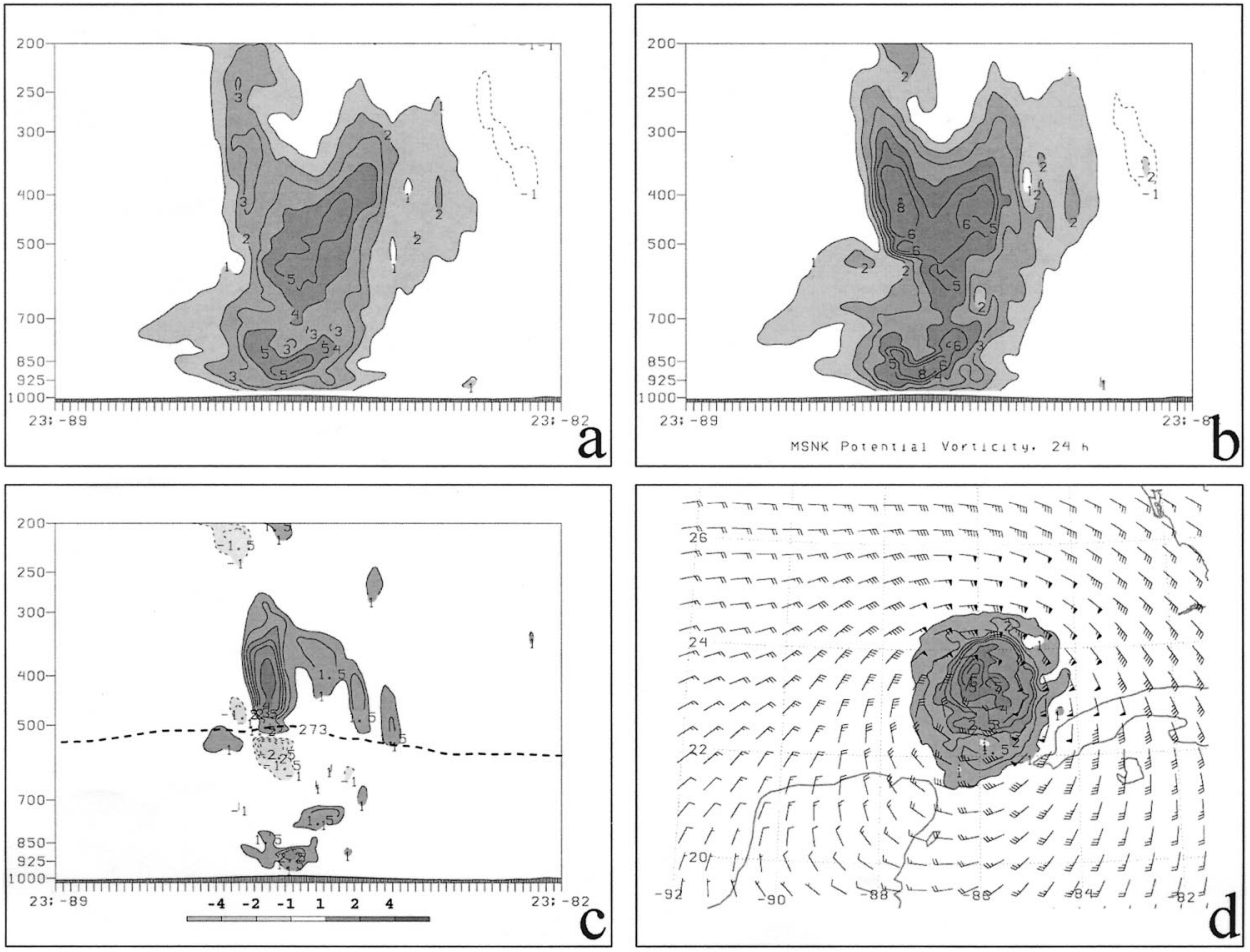

FIG. 16. Potential vorticity difference summary for 24-h simulations valid at 0000 UTC 2 Oct: (a) Ertel potential vorticity cross section (PVU, contoured and shaded) for CTRL simulation; (b) as in (a) except for MSNK simulation; (c) difference (MSNK - CTRL) potential vorticity (PVU, contoured and shaded as indicated in legend), and 273-K isotherm (thick dashed line); and (d) 850-700-hPa layer-averaged PV and 800-hPa winds from CTRL simulation. 

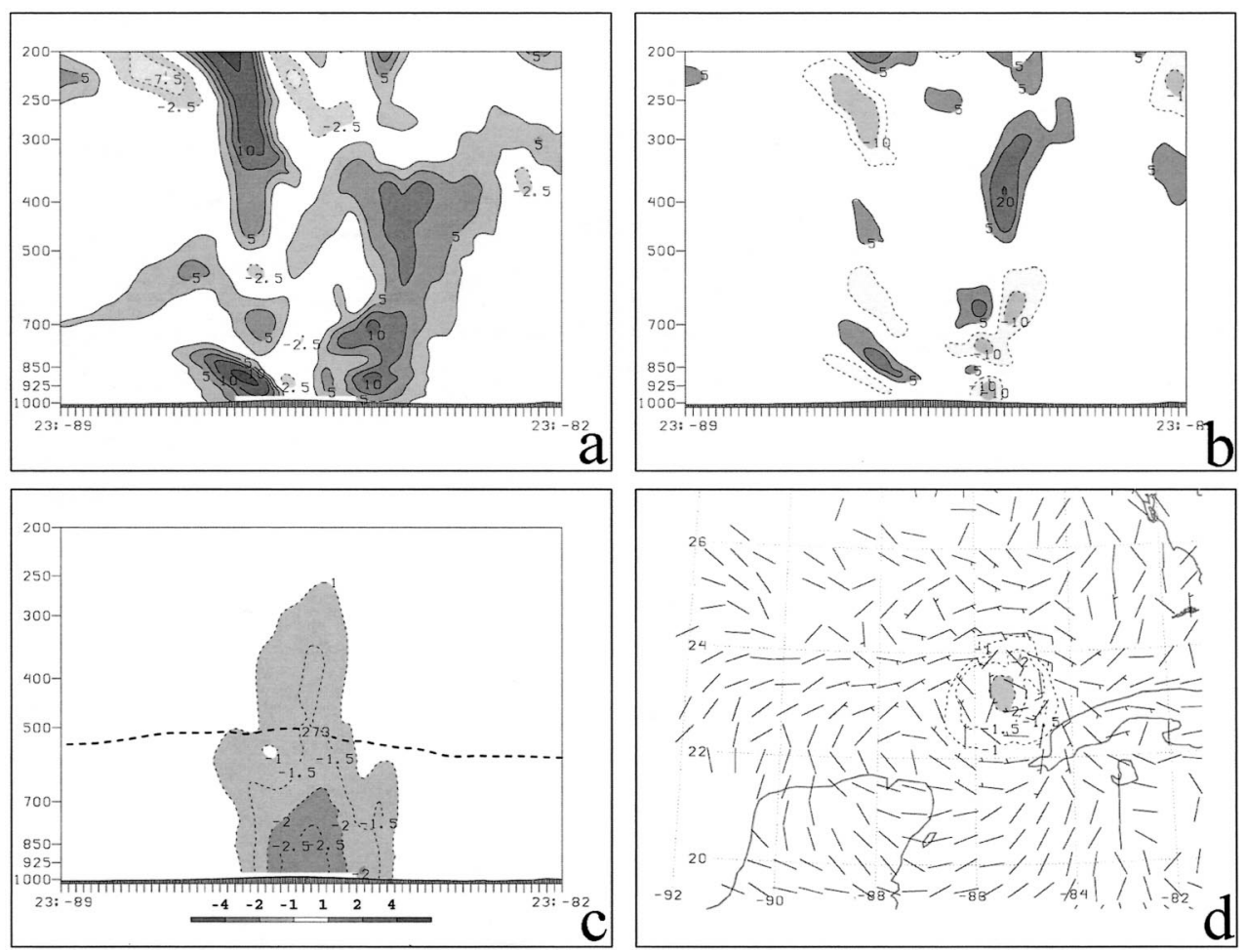

FIG. 17. Additional cross-sectional difference fields for 24-h simulations: (a) difference (MSNK - CTRL) in wind speed (kt, contoured and shaded); (b) as in (a) except for divergence $\left(\times 10^{-5} \mathrm{~s}^{-1}\right.$, contoured and shaded); (c) as in (a) except for geopotential height difference (contoured and shaded as in legend); and (d) 700-hPa geopotential height difference (MSNK - CTRL) and vector difference 700-hPa winds.

\section{Conclusions and implications for future research}

Pressure changes due to sources and sinks of atmospheric mass resulting from evaporation and precipitation are currently neglected in most meteorological applications. The pervasive use of this assumption has received some attention in the literature, but there appears to have been little or no discussion of the viability of this mechanism as an important factor in the dynamics of heavily precipitating systems. Here, we examined the validity of this assumption for the case of tropical cyclones, in which precipitation rates are sufficient to provide a pressure-equivalent mass reduction on the order of $25 \mathrm{hPa} \mathrm{day}^{-1}$ or more. The hypothesis tested here is that the precipitation mass sink provides a nonnegligible contribution to the dynamics of heavily precipitating storms through modification of the mass field and resulting motion adjustments. A secondary hypothesis is that the inclusion of the mass sink effect in numerical models will result in stronger cyclonic systems that are characterized by heavier precipitation.

Three initial hypothesis tests were presented using the case of Hurricane Lili (2002). An MM5 simulation was used as a high-resolution dataset from which to compute mass and PV budgets. MM5 provided a reasonable representation of Lili, including an eye structure and realistic precipitation and central-pressure deepen- ing rates. Computation of a simple mass budget for the model storm demonstrates that the mass loss due to precipitation exceeded that needed to explain the net hydrostatic pressure decrease within a $100-\mathrm{km}$ radius of the storm center. Although the tropical cyclone environment is characterized by cancellation between strong convergent inflow in the lower troposphere and strong divergent outflow aloft, the precipitation mass sink contribution to the storm-average mass budget is not negligible.

The mass sink effect can alter the PV through the concentration of PV substance in isentropic water vapor source layers. Using the framework of Ooyama (2001) and Schubert et al. (2001), the mass sink contribution to the PV tendency computed for the Lili case was found to provide a consistent positive PV tendency of approximately $10 \mathrm{PVU}$ day $^{-1}$ near and beneath the freezing level. Relative to the diabatic PV tendency, the magnitude of the local contribution from the mass sink was very small; however, the diabatic PV-tendency term exhibits strong cancellation whereas the mass sink tendency is consistently positive. Comparison of the PV field in two Eta Model simulations, one of which included mass sink effects, demonstrates that the hurricane PV tower is stronger in the mass sink simulation, especially above the freezing level.

The most comprehensive test of the hypothesis was 
based on comparison of two numerical simulations from the workstation version of the Eta Model. In the control simulation, a modest deepening of Lili was obtained. Differences between the control simulation and an experimental simulation (MSNK) based on a version of the model in which precipitation mass sink effects were taken into account are consistent with the hypothesis that the mass sink effect is not negligible. Specifically, the MSNK simulation produced a storm that was 2-5 $\mathrm{hPa}$ deeper, characterized by a cyclonic flow that was typically 5-15 kt stronger at the 10-m level and accompanied by heavier precipitation that more completely encircled the eye. Although these differences suggest that the mass sink effect is not a dominant influence, the Eta CTRL simulation only deepened Lili about 14 $\mathrm{hPa}$ during the 48-h integration, meaning that the additional deepening in MSNK represents a 30\% increase beyond CTRL. The sea level pressure differences became pronounced within the first $24 \mathrm{~h}$ of the simulation and did not amplify significantly beyond about $30 \mathrm{~h}$, while differences in wind speed and precipitation continued to amplify until the end of the model integration. While the air-sea interaction instability theory of Emanuel (1986) explains the basic mechanism of tropical cyclogenesis, the precipitation mass sink may provide an additional physical development mechanism that contributes to the intensity of the system in a manner that is consistent with the air-sea interaction process.

The mass sink mechanism may be important in other precipitation systems, including midlatitude convection, extratropical cyclones, and heavy orographic rainfall. The fact that the precipitation mass flux divergence is large near the melting layer indicates that the mass sink mechanism would reinforce the positive PV tendencies due to melting effects during the formation of midlevel convectively generated vortices (MCVs; Davis and Trier 2002). The relative importance of these effects remains for future study. NWP models have difficulty in simulating organized, propagating convection (e.g., Carbone et al. 2002; Davis et al. 2002); it is possible that inclusion of the mass sink mechanism could improve this situation.

Future research will examine the relative importance and interdependence of the precipitation mass sink and water-loading effects in both hydrostatic and nonhydrostatic models. Kato (1997) has published results indicating that accounting for water-loading effects in hydrostatic models was more important than inclusion of nonhydrostatic effects for $10-20-\mathrm{km}$ grid spacing. Although additional modeling experiments are needed, it is safe to conclude that the representation of precipitation systems in models that do not account for the mass sink will be distorted by the burden of additional mass exhaust that nature removes to the surface via precipitation. As evident from the equations of Ooyama (2001), the mass sink also appears explicitly in the momentum equation (see also Qiu et al. 1993); momentum and entropy transfer from falling precipitation may also represent important processes and should ultimately be included in operational NWP models as well.

Acknowledgments. We are greatly indebted to Fedor Mesinger of NCEP for his enthusiastic support during this research and, with Dusan Jovic (NCEP), for providing code modifications necessary to set up a "mass sink" version of the workstation Eta Model. We also thank Matthew Pyle of NCEP for providing initial condition data for the Lili case and for assistance in setting up the workstation Eta. Brad Ferrier (NCEP) and Fedor Mesinger provided helpful suggestions regarding the Eta simulations of Lili and insightful discussions concerning model representation of precipitation processes. Jimy Dudhia (NCAR), Kerry Emanuel (MIT), Mark Stoelinga (University of Washington), Michael Brennan (North Carolina State), and two anonymous reviewers provided constructive comments during the preparation of this manuscript. We thank Jimy Dudhia, Jack Kain, and Michael Brennan for their assistance with modifications to the MM5 model to output individual heating terms. This research was supported by NSF Grants ATM-0079425 and ATM-0334427, awarded to North Carolina State University, and an American Meteorological Society Graduate Fellowship awarded to the second author. The PSU-NCAR MM5 Model was made available through NCAR, sponsored by the NSF, and the workstation version of the Eta Model was made available by NCEP. Other data and imagery were provided by NCEP, the Unidata program, the Tropical Prediction Center, and the Naval Research Laboratory.

\section{REFERENCES}

Anthes, R. A., 1982: Tropical Cyclones: Their Evolution, Structure, and Effects. Amer. Meteor. Soc., 208 pp.

Braun, S. A., 2002: A cloud-resolving simulation of Hurricane Bob (1991): Storm structure and eyewall buoyancy. Mon. Wea. Rev., 130, $1573-1592$.

__ , and W.-K. Tao, 2000: Sensitivity of high-resolution simulations of Hurricane Bob (1991) to planetary boundary layer parameterizations. Mon. Wea. Rev., 128, 3941-3961.

Carbone, R. E., J. D. Tuttle, D. A. Ahijevych, and S. B. Trier, 2002: Inferences of predictability associated with warm season precipitation episodes. J. Atmos. Sci., 59, 2033-2056.

Charney, J. G., and A. Eliassen, 1964: On the growth of the hurricane depression. J. Atmos. Sci., 21, 68-75.

Davies, T., M. Diamantakis, and A. J. Malcom, 2002: Moist NWP equations and missing terms. Preprints, 15th Conf. on Numerical Weather Prediction, San Antonio, TX, Amer. Meteor. Soc., 4142.

Davis, C. A., and L. F. Bosart, 2001: Numerical simulations of the genesis of Hurricane Diana (1984). Part I: Control simulation. Mon. Wea. Rev., 129, 1859-1881.

— , and — 2002: Numerical simulations of the genesis of Hurricane Diana (1984). Part II: Sensitivity of track and intensity prediction. Mon. Wea. Rev., 130, 1100-1124.

__ and S. B. Trier, 2002: Cloud-resolving simulations of mesoscale vortex intensification and its effect on a serial mesoscale convective system. Mon. Wea. Rev., 130, 2839-2858.

, D. A. Ahijevich, R. E. Carbone, K. W. Manning, and J. Tuttle, 2002: Statistical-dynamical forecasts of warm season rainfall over North America. Preprints, 19th Conf. on Weather Analysis 
and Forecasting, San Antonio, TX, Amer. Meteor. Soc., 152155.

Dudhia, J., 1993: A nonhydrostatic version of the Penn State-NCAR Mesoscale Model: Validation tests and simulations of an Atlantic cyclone and cold front. Mon. Wea. Rev., 121, 1493-1513.

Dutton, J. A., 1986: The Ceaseless Wind. Dover, 615 pp.

Emanuel, K. A., 1986: An air-sea interaction theory for tropical cyclones. Part I: Steady-state maintenance. J. Atmos. Sci., 43, 585-604.

— M. Fantini, and A. J. Thorpe, 1987: Baroclinic instability in an environment of small stability to slantwise moist convection. Part I: Two-dimensional models. J. Atmos. Sci., 44, 1559-1573.

Grell, G., J. Dudhia, and D. Stauffer, 1994: A description of the fifthgeneration Penn State/NCAR Mesoscale Model (MM5). NCAR Tech. Note NCAR/TN-398 + STR, 117 pp.

Gu, H., and Z. Qian, 1991: A discussion about the role of the water vapor source/sink term in continuity equation of numerical models. Chin. Sci. Bull., 36, 16-21.

Haynes, P. H., and M. E. McIntyre, 1987: On the evolution of vorticity and potential vorticity in the presence of diabatic heating and frictional or other forces. J. Atmos. Sci., 44, 828-841.

Kato, T., 1997: Hydrostatic and non-hydrostatic simulations of moist convection: Review and further study. Meteor. Atmos. Phys., 63, $39-51$.

Keyser, D. A., and D. R. Johnson, 1984: Effects of diabatic heating on the ageostrophic circulation of an upper tropospheric jet streak. Mon. Wea. Rev., 112, 1709-1724.

Kurihara, Y., M. A. Bender, and R. J. Ross, 1993: An initialization scheme of hurricane models by vortex specification. Mon. Wea. Rev., 121, 2030-2045.

R. E. Tuleya, and R. J. Ross, 1995: Improvements in the GFDL hurricane prediction system. Mon. Wea. Rev., 123, 27912801.

Lawrence, M. B., cited 2003: National Hurricane Center tropical cyclone report. [Available online at http://www.nhc.noaa.gov/ 2002lili.shtml.]

Lin, Y.-L., R. D. Farley, and H. D. Orville, 1983: Bulk parameterization of the snow field in a cloud model. J. Climate Appl. Meteor., 22, 1065-1092.

Liu, Q., T. Marchok, H.-L. Pan, M. A. Bender, and S. Lord, cited 2000: Improvements in hurricane initialization and forecasting at NCEP with global and regional (GFDL) models. [Available online at http://www.nws.noaa.gov/om/tpb/472.pdf.]

Mesinger, F., 1984: A blocking technique for representation of mountains in atmospheric models. Riv. Meteor. Aeronaut., 44, 195202.

_ 1998: Performance of the 48-km Eta in forecasting tracks of major landfalling Atlantic hurricanes of the 1996 season, Bertha and Fran. Preprints, 16th Conf. on Weather Analysis and Forecasting, Phoenix, AZ, Amer. Meteor. Soc., 526-528.

— Z. Z. Janjic, S. Nickovic, D. Gavrilov, and D. G. Deaven, 1988: The step-mountain coordinate: Model description, and perfor- mance for cases of Alpine lee cyclogenesis and for a case of an Appalachian redevelopment. Mon. Wea. Rev., 116, 1493-1518.

Möller, J. D., and R. K. Smith, 1994: The development of potential vorticity in a hurricane-like vortex. Quart. J. Roy. Meteor. Soc. 120, $1255-1265$

Ooyama, K. V., 1982: Conceptual evolution of the theory and modeling of the tropical cyclone. J. Meteor. Soc. Japan, 60, 369379.

— - 1990: A thermodynamic foundation for modeling the moist atmosphere. J. Atmos. Sci., 47, 2580-2593.

- 2001: A dynamic and thermodynamic foundation for modeling the moist atmosphere with parameterized microphysics. $J$. At mos. Sci., 58, 2073-2102.

Palmén, E., and H. Riehl, 1957: Budget of angular momentum and energy in tropical cyclones. J. Meteor., 14, 150-159.

Pu, Z.-X., and S. A. Braun, 2001: Evaluation of bogus vortex techniques with four-dimensional variational data assimilation. Mon Wea. Rev., 129, 2023-2039.

Qiu, C.-J., J.-W. Bao, and Q. Xu, 1991: The significance of mass sink due to precipitation. Preprints, 9th Conf. on Mesoscale Processes, Denver, CO, Amer. Meteor. Soc., 364-365.

,$- \ldots$, and -1993 : Is the mass sink due to precipitation negligible? Mon. Wea. Rev., 121, 853-857.

Savijärvi, H., 1995: Water mass forcing. Bietr. Phys. Atmos., 68, $75-$ 84

Schubert, W. H., and B. T. Alworth, 1987: Evolution of potential vorticity in tropical cyclones. Quart. J. Roy. Meteor. Soc., 113, $147-162$

— S. A. Hausman, M. Garcia, K. V. Ooyama, and H.-C. Kuo, 2001: Potential vorticity in a moist atmosphere. J. Atmos. Sci., $\mathbf{5 8}, 3148-3157$.

Stoelinga, M. T., 1996: A potential vorticity-based study of the role of diabatic heating and friction in a numerically simulated baroclinic cyclone. Mon. Wea. Rev., 124, 849-874.

Sutcliffe, R. C., 1947: A contribution to the problem of development. Quart. J. Roy. Meteor. Soc., 73, 370-383.

Trenberth, K. E., 1991: Climate diagnostics from global analyses: Conservation of mass in ECMWF analyses. J. Climate, 4, 707722.

— J. J. R. Christy, and J. G. Olson, 1987: Global atmospheric mass, surface pressure, and water vapor variations. J. Geophys. Res., 92, $14815-14826$.

Van den Dool, H. M., and S. Saha, 1993: Seasonal redistribution and conservation of atmospheric mass in a general circulation model. J. Climate, 6, 22-30.

Zhang, D.-L., Y. Liu, and M. K. Yau, 2000: A multiscale numerical study of Hurricane Andrew (1992). Part III: Dynamically induced vertical motion. Mon. Wea. Rev., 128, 3772-3788.

,$- \ldots$, and -2001 : A multiscale numerical study of Hurricane Andrew (1992). Part IV: Unbalanced flows. Mon. Wea Rev., 129, 92-107.

$\longrightarrow$ - — and $\longrightarrow$, 2002: A multiscale numerical study of Hurricane Andrew (1992). Part V: Inner-core thermodynamics. Mon. Wea. Rev., 130, 2745-2763. 